\title{
The Moduli Space of Kähler Structures on a Real Compact Symplectic Manifold
}

By

\author{
Akira FUJIKI* and Georg SCHUMACHER**
}

\section{Introduction}

Let $(M, \omega)$ be a compact symplectic $C^{\infty}$-manifold. We study in this paper the moduli space $\mathscr{M}_{\omega}$ of $C^{\infty}$ Kähler structures on $(M, \omega)$. These are precisely the complex structures $J$ on $M$ such that $\omega$ is a Kähler form on the complex manifold $(M, J)$.

First $\mathscr{M}_{\omega}$ can be naturally described as the quotient space $\mathscr{C}_{i, \omega} / \mathscr{D}_{\omega}$ of the space $\mathscr{C}_{i, \omega}$ of Kähler structures on $(M, \omega)$ by the action of the groups $\mathscr{D}_{\omega}$ of symplectic diffeomorphisms on $(M, \omega)$. In order to treat this infinite dimensional object, we introduce the Sobolev $H^{k}$-completions $\mathscr{C}_{i, \omega}^{k}$ and $\mathscr{D}_{\omega}^{k}$ of $\mathscr{C}_{i, \omega}$ and $\mathscr{D}_{\omega}$ respectively and consider the quotient spaces $\mathscr{M}_{\omega}^{k}:=\mathscr{C}_{i, \omega}^{k} / \mathscr{D}_{\omega}^{k+1}$ for all sufficiently large $k$. Therefore the spaces $\mathscr{M}_{\omega}^{k}$ are quotients of complex Hilbert analytic spaces by topological groups which are real Hilbert manifolds. We prove that these actions are proper (Theorem 3.3). We view $\mathscr{M}_{\omega}$ as the inverse limit of $\mathscr{M}_{\omega}^{k}$ with the induced Hausdorff topology. It contains the open subspace $\mathscr{M}_{\omega}^{\prime}$ of Kähler structures which admit no nonvanishing holomorphic vector fields. Our main result (Theorem 6.9) states that $\mathscr{M}_{\omega}^{\prime}=\lim \mathscr{M}_{\omega}^{k^{\prime}}$ is naturally an ILH-V-space, i。 e. $\mathscr{M}_{\omega}^{\prime}$ is locally a quotient of an ILH-space by a finite group. Here an ILH-space is essentially an ILH-manifold in the sense of Omori [OM] with singularities.

The proof of the main theorem is mainly based upon the construction of slices in the ILH-category for the action of $\mathscr{D}_{\omega}$ on $\mathscr{C}_{i, \omega}$

Communicated by K. Saito, August 12, 1987.

* Yoshida college, Kyoto University, Kyoto 606, Japan.

** Mathematisches Institut der Universität, Einsteinstraße 62, D-4400 Münster, West Germany. Supported by DFG (Heisenberg-Programm) 
(Theorem 5.6). Namely we construct for each point $\sigma$ of $\mathscr{C}_{i, \infty}$ a locally closed subspace $\mathscr{S}$ passing through $\sigma$ and an ILH-diffeomorphism of $V \times \mathscr{S}$ to a neighborhood of $\sigma$ in $\mathscr{C}_{i, \omega}$, where $V$ is a neighborhood of $\sigma$ in its orbit under $\mathscr{D}_{\omega}$. Moreover $\mathscr{S}$ is invariant under the action of the isotropy group $\mathscr{D}_{\sigma}$ of $\sigma$, and if $\varphi \cdot \mathscr{S} \cap \mathscr{S} \neq \emptyset$ for a $\varphi \in \mathscr{D}_{\omega}$ then $\varphi \in \mathscr{D}_{\sigma}$. Note that $\mathscr{D}_{\sigma}$ is just the group of holomorphic isometries of the corresponding Kähler structure. The quotient maps $\mathscr{S} \rightarrow \mathscr{S} / \mathscr{D}_{0}$ for various $\sigma$ induce the local uniformizing systems for our ILH-V-structure. The construction of the above slices requires an infinitesimal slice theorem, which states the decomposition of the space of $(0,2)$-tensors on $(M, \sigma)$ into the tangent space of $\mathscr{D}_{\omega}^{k+1}$ and its orthogonal complement by means of a differential operator with injective symbol (Theorem 4.3). The above slice theorem is an analogue of a result of Ebin [EB], who constructs a slice for the action of the diffeomorphism group of the space of Riemannian metrics on a fixed compact $C^{\infty}$ manifold (cf. also [KO]).

Let $\lambda \in H^{2}(M, R)$ be the de Rham class of $\omega$ and $\mathscr{M}_{\lambda}$ the set of isomorphism classes of polarized Kähler manifolds whose underlying differentiable structure is $(M, \lambda)[\mathrm{FU}, \mathrm{SCH}]$. Then we have a natural map from $\mathscr{M}_{\omega}$ to $\mathscr{M}_{\lambda}$. We show that $\mathscr{M}_{\lambda}$ is a disjoint union of the images of $\mathscr{M}_{\omega}$, where $\omega$ runs through a set of representatives of the space of connected components of the symplectic forms representing $\lambda$. In particular $\mathscr{M}_{\lambda}$ has countable topology.

The essential part of this work has been done, while the second named author stayed at RIMS, Kyoto University. He would like to express his gratitude for its kind hospitality and for support by DFG. The first named author expresses his gratitude towards the Münster university for its kind hospitality.

\section{Almost Complex Structures on a Symplectic Manifold}

Let $M$ be a fixed (connected) compact $C^{\infty}$-manifold of even dimension $2 m$, and $T$ its tangent bundle. An almost complex structure on $M$ is by definition an endomorphism $\sigma$ of $T$ with $\sigma^{2}=i d_{T}$. If $\sigma_{x}: T_{x}^{c} \rightarrow T_{x}^{c}, x \in M$, is the induced endomorphism on a fiber of the complexified tangent bundle, then the eigenspaces $S_{\sigma, x}$ with eigenspace $-\sqrt{-1}$ form a subbundle $S_{o} \subset T^{c}$ such that $S_{o} \oplus \bar{S}_{\sigma}=T^{c}$, where the 
bar denotes the conjugation on $\mathbb{C}$. Conversely such a subbundle determines an almost complex structure.

If $M$ is equipped with an arbitrary $C^{\infty}$-metric, then $H^{k}(M)$ shall denote the Sobolev space of real functions on $M$ whose distributional covariant derivatives exist up to order $k$ and are square-integrable. For any coordinate covering of $M$ and a subordinate partition of unity $\left\{\alpha_{i}\right\}$, the space $H^{k}(M)$ consists of all functions $f$ such that $\alpha_{i} \cdot f \in H^{k}\left(\mathbb{R}^{2 m}\right)$. Further given a (normed) vector bundle $E$ on $M$ of class $C^{\infty}$, we denote by $H^{k}(E)$ the space of all $H^{k}$-sections. The space of $H^{k}$-sections of a vector bundle is well-defined provided its transition functions can be chosen of class $H^{k}$ for $m \leqq k \leqq \infty$. A subbundle $F \subset E$ is called of class $H^{k}$, if it can be spanned locally by $H^{k}$-sections. In this case we have a natural embedding $H^{k}(F) \rightarrow H^{k}(E)$ as a closed subspace.

Definition 1.1. For any natural number $k>m$ we set $\mathscr{C}_{a}^{k}:=\left\{S \subset T^{c} ; S\right.$ is an $H^{k}$ subbundle, $\left.S \oplus \bar{S}=T^{c}\right\}$.

The elements of $\mathscr{C}_{a}^{k}$ will be called almost complex structures of class $H^{k}$. Two almost complex structures $\sigma, \tau$ will be called of finite distance, if the projection $S_{\sigma} \oplus \bar{S}_{\sigma} \rightarrow \bar{S}_{\sigma}$, restricted to $S_{\tau}$, is an isomorphism of $H^{k}$ vector bundles. If $\sigma \in \mathscr{C}_{a}^{k}$, then

$$
U(\sigma):=\left\{\tau \in \mathscr{C}_{a}^{k} ; \sigma \text { and } \tau \text { of finite distance }\right\}
$$

Proposition 1.2. Let $m<k<\infty$. Then:

(i) The space $\mathscr{C}_{a}^{k}$ carries the structure of a complex analytic Hilbert manifold, which is Hausdorff (cf. [DOU]).

(ii) If $\sigma \in \mathscr{C}_{a}^{k}$, then there is a natural $\mathbb{C}$-analytic isomorphism of manifolds between $U(\sigma)$ and an open subset of the tangent space $T_{\sigma}\left(\mathscr{C}_{a}^{k}\right)$ $\simeq H^{k}\left(S_{\sigma}^{*} \otimes \bar{S}_{\sigma}\right)$, where $S_{o}^{*}$ denotes the dual of $S_{\sigma}$.

Proof of (i). We shall use the transition functions later and therefore give a short argument.

Let $\tau \in U(\sigma)$. Then $S_{\tau}$ is the graph of a map $\left(i d_{S_{\sigma}}-A\right): S_{\sigma} \rightarrow \bar{S}_{\sigma}$, where $A \in H^{k}\left(S_{\sigma}^{*} \otimes \bar{S}_{\sigma}\right)$ is uniquely determined. We use for this proof the notation $A=A_{\sigma}(\tau)$ and claim that the natural bijection $\phi$ sending $A_{\sigma}(\rho)$ to $A_{\tau}(\rho), \rho \in U(\sigma) \cap U(\tau)$ is $\mathbb{C}$-analytic, which can be reduced 
to the case $\tau \in U(\sigma)$. We fix a point $p$ of $M$ and write $S_{\rho}$ and $A_{\tau}(\rho)$ for $S_{\rho, p}$ and $A_{\tau}(\rho)_{p}$. Any point of $S_{\rho}$ has unique representations $x-A_{\sigma}(\rho)(x)=y-A_{\tau}(\rho)(y)$ in $S_{\sigma} \oplus \bar{S}_{\sigma}$ and $S_{\tau} \oplus \bar{S}_{\tau}$; furthermore

$$
x^{\prime}=x-\overline{A_{\sigma}(\tau)} A_{\sigma}(\rho)(x) \in S_{\sigma} .
$$

As

$$
x^{\prime}=y-\left(A_{\tau}(\rho)(y)-A_{\sigma}(\rho)(x)+\overline{A_{\sigma}(\tau)} A_{\sigma}(\rho)(x)\right) \in S_{\tau} \oplus \bar{S}_{\tau}
$$

is a decomposition $A_{\tau}(\sigma)(y)=A_{\tau}(\rho)(y)-A_{\sigma}(\rho)(x)+\overline{A_{\sigma}(\tau)} A_{\sigma}(\rho)(x)$ and $\quad x^{\prime}=y-A_{\tau}(\sigma)(y)=x-\overline{A_{\sigma}(\tau)} A_{\sigma}(\rho)(x)$. Denote by $I_{\sigma}: S_{\sigma} \rightarrow S_{\sigma} \oplus \overline{S_{\sigma}}$ etc. the canonical map. Then $I_{\sigma}-\overline{A_{\sigma}(\tau)} A_{\sigma}(\rho): S_{\sigma} \rightarrow S_{\sigma}$ is by the above equation ( $p \in M$ fixed) injective, and $\left(I_{\sigma}-\overline{A_{\sigma}(\tau)} A_{\sigma}(\rho)\right)^{-1} \in H^{k}\left(S_{\sigma}^{*} \oplus \bar{S}_{\sigma}\right)$. The $\boldsymbol{C}$-analyticity of $\phi$ follows from:

$$
A_{\tau}(\rho)=I_{\tau}-\left(I_{\sigma}-A_{\sigma}(\rho)\right)\left(I_{\sigma}-\overline{A_{\sigma}(\tau)} A_{\sigma}(\rho)\right)^{-1}\left(I_{\tau}-A_{\tau}(\sigma)\right) .
$$

Given two different $H^{k}$ sections of $S_{\sigma}^{*} \otimes \bar{S}_{\sigma}$, their values are different at some point of $M$. Since the evaluation map is continuous, the Hausdorff property follows immediately.

Definition 1.3. We set $\mathscr{C}_{i}^{k}=\left\{\sigma \in \mathscr{C}_{a}^{k} ; \sigma\right.$ is an integrable almost complex structure\}. A complex analytic subspace of a complex analytic Hilbert manifold is by definition a subspace which is locally the zerolocus of a holomorphic map to a Hilbert space.

Proposition 1.4. The set $\mathscr{C}_{i}^{k}$ is a closed complex analytic subspace of $\mathscr{C}_{a}^{k}$ of infinite dimension.

Proof. Let $\sigma \in \mathscr{C}_{{ }_{i \circ}^{k}}^{k}$ Let $T^{(0,1)}=S_{\sigma}$ be the bundle of tangent $(0,1)$ vectors with respect to $\sigma$. Then one assigns to any $\rho \in U(\sigma)$ the tensor $A \in H^{k}\left(S_{\sigma}^{*} \otimes \bar{S}_{\sigma}\right)$ as in 1.2 , which induces a $(0,1)$-form $\phi$ with values in $T^{(1,0)}$. The integrability condition is

$$
\bar{\partial} \phi-\frac{1}{2}[\phi, \phi]=0 \text {. }
$$

The corresponding map $\left.H^{k}\left(S_{\sigma}^{*} \otimes \bar{S}_{\sigma}\right) \rightarrow H^{k-1}\left(\Lambda^{2}\left(S_{\sigma}^{*}\right) \otimes \bar{S}_{\sigma}\right)\right)$ is a quadratic polynomial with necessary estimates. So $\mathscr{C}_{i}^{k} \cap U(\sigma)$ is $\mathbb{C}$-analytic in $U(\sigma)$.

For the closedness of $\mathscr{C}_{i}^{k}$ in $\mathscr{C}_{a}^{k}$, we pick a sequence of integrable structures $\sigma_{n}$ converging to $\tau \in \mathscr{C}_{a}^{k}$. Now there exists an $n_{0}$ such that 
all $\sigma_{n}$ are of finite distance of $\sigma_{n_{0}} n \geqq n_{0}$, which yields the closedness.

Let $\omega$ be a (real) symplectic form of class $C^{\infty}$ on $M_{j}$ i. $_{0}$ e. a nowhere degenerate real $d$-closed 2-form on $M$.

Definition 1 .5. We set

$\mathscr{C}_{a, \omega}^{k}:=\left\{\sigma \in \mathscr{C}_{a}^{k} ; \omega\right.$ is $\sigma$-invariant and induces a positive definite Hermitian form on $S_{\sigma}$ \}

and

$$
\mathscr{C}^{k}{ }_{i, \omega}:=\mathscr{C}_{a, \omega}^{k} \cap \mathscr{C}_{i}^{k} .
$$

(We identify $\omega$ with its $\mathbb{C}$ linear extension to $T^{C}$.)

Any element $\sigma \in \mathscr{C}_{a, \omega}^{k}\left(\right.$ resp. $\left.\mathscr{C}_{i, \omega}^{k}\right)$ is called an almost Kähler (resp. Kähler) structure on the real symplectic manifold $(M, \omega)$.

For all $\sigma \in \mathscr{C}_{a, \omega}^{k}$ the symplectic form $\omega$ furnishes a canonical isomorphism between $S_{\sigma}^{*}$ and $\bar{S}_{\sigma}$ and an isomorphism $\iota: H^{k}\left(S_{\sigma}^{*} \otimes \bar{S}_{\sigma}\right) \rightarrow$ $H^{k}\left(\bar{S}_{o} \otimes \bar{S}_{\sigma}\right)$.

Proposition 1.6. (i) Let $\sigma \in \mathscr{C}_{a, \omega}^{k}$. Then $\mathscr{C}_{a, \omega}^{k}$ is contained in the coordinate neighborhood $U(\sigma) \subset \mathscr{C}_{a}^{k}$, and $\mathscr{C}_{a, \omega}^{k}$ is a locally closed submanifold of $U(\sigma)$.

(ii) The natural open embedding of $U(\sigma)$ into its tangent space $T_{\sigma}\left(\mathscr{C}_{a}^{k}\right)$ induces an open embedding of $\mathscr{C}_{a, \infty}^{k}$ into its tangent space $T_{\sigma}\left(\mathscr{C}_{a, \omega}^{k}\right)$. Moreover with respect to $T_{\sigma}\left(\mathscr{C}_{a}^{k}\right) \simeq H^{k}\left(\bar{S}_{\sigma} \otimes \bar{S}_{\sigma}\right)$ (via $\left.\iota\right) T_{\sigma}\left(\mathscr{C}_{a, \omega}^{k}\right)$ corresponds to $H^{k}\left(S^{2}\left(\bar{S}_{\sigma}\right)\right)$.

(iii) $\mathscr{C}_{a, \infty}^{k}$ is contractible.

We prove (i) and (ii): Let $\left(v_{1}, \ldots, v_{m}\right)$ be a local $m$-frame of class $H^{k}$ that spans $S_{\sigma o}$. Take any $\tau \in \mathscr{C}_{a, \omega}^{k}$ such that $S_{\tau}$ is spanned by a local $m$-frame $\left(w_{1}, \ldots, w_{m}\right)$ of class $H^{k}$. Let

$$
w_{i}=A_{\imath}^{k} v_{k}-A_{i}^{i} v_{i}
$$

with complex $H^{k}$ coefficients. Since $\omega$ induces a positive definite Hermitian form on $S_{\tau}$ and $\tau \in U(\sigma),\left(A_{t}^{k}\right)_{i, k=1, \ldots m}$ is invertible and therefore by passing to another local frame $\left(w_{1}, \ldots, w_{m}\right)$ we may assume $A_{i}^{k}=\delta_{i}^{k}$. By an elementary calculation one can see that $v_{i} \rightarrow A_{i}^{i} v_{i}$ defines an $H^{k}$-section $A$ of $S_{o}^{*} \otimes \bar{S}_{\sigma}$ which corresponds to $\tau$ in the sense of 1.2. By the isomorphism $S_{\sigma}^{*} \otimes \bar{S}_{\sigma} \rightarrow \bar{S}_{\sigma} \otimes \bar{S}_{\sigma}$ induced by $\omega$, $A$ is mapped to a symmetric tensor. The image of $\mathscr{C}_{a, \omega}^{k}$ in $U(\sigma)$ is 
characterized by symmetricity and by the condition that $I d_{S_{\sigma}}-\bar{A} \circ A$ is positive definite with respect to the Hermitian structure induced by $\sigma$ and $\omega_{0}$. (iii) is a consequence from the above description of elements of $\mathscr{C}_{a, \omega}^{k}$ by sections $A \in H^{k}\left(S_{\sigma}^{*} \otimes \bar{S}_{\sigma}\right)$.

Remark 1.7. One can show that $\mathscr{C}_{a, \omega}^{k}$ is diffeomorphic to a Hilbert space.

Remark 1.8. Let $\mathscr{G}_{x} \rightarrow M$ be the $C^{\infty}$ fiber bundle whose fibers consist of the Graßmann manifolds of $m$-planes in $T_{x}^{c}$ for $x \in M$.

We denote by $\mathscr{A}=\bigcup_{x \in M} \mathscr{A}_{x} \subset \mathscr{G}_{x}$ the open subspace given by $\mathscr{A}_{x}=$ $\left\{S_{x} \in \mathscr{G}_{x_{x}} ; S_{x} \cap \bar{S}_{x}=(0)\right\}$ and by $\mathscr{B} \rightarrow M$ the fiber bundle with fibers $\mathscr{B}_{x}=\left\{S_{x} \in \mathscr{A}_{x} ; \omega_{x}\right.$ induces a positive definite Hermitian structure on $\left.\bar{S}_{x}\right\}$.

(Note that the fibers $\mathscr{B}_{x}$ are canonically isomorphic to Siegel upper half spaces of dimension $m(m+1) / 2$.)

Then $\mathscr{C}_{a}^{k}$ and $\mathscr{C}_{a, \omega}^{k}$ resp. are the spaces of $H^{k}$-sections of $\mathscr{A} \rightarrow M$ and $\mathscr{B} \rightarrow M$ resp. in the sense of Palais [PA].

We shall construct distinguished Sobolev norms on the tangent spaces of $\mathscr{C}_{a, \omega}^{k}$.

Let $\sigma \in \mathscr{C}_{a, \omega}^{k}$. Denote by $g_{\sigma}$ the Riemannian metric on $M$ underlying the Hermitian structure induced by $\sigma$ and $\omega$; more precisely

$$
g_{\sigma}(\xi, \eta)=-\omega(\xi, \sigma \eta)
$$

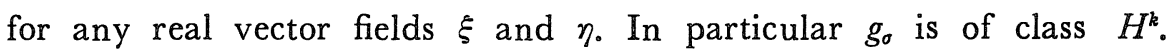
The symmetric tensor $g_{o}$ turns $S^{2}\left(\bar{S}_{\sigma}\right)$ into a Hermitian vector bundle. On the space of $H^{k}$-sections of $S^{2}\left(\bar{S}_{\sigma}\right)$ i. e. on $T_{\sigma}\left(\mathscr{C}_{a, \omega}^{k}\right)$ a weak (i. e. non-complete) inner product is defined by

$$
(\xi, \eta)_{0, \sigma}:=\int_{M} g_{\sigma}(\xi, \eta)(x) \omega^{m} .
$$

Observe that by means of the natural isomorphism (cf. 1.6) $T_{\sigma}\left(\mathscr{C}_{a, \omega}^{k}\right) \rightarrow H^{k}\left(S_{\sigma}^{*} \otimes \bar{S}_{\sigma}\right)$ we have $g_{\sigma}(\xi, \eta)=$ trace $(\bar{\eta} \cdot \xi)$. In 1.7 the fibers $\mathscr{B}_{x}$ are realized as bounded symmetric domains which we equip with the Bergman metric $m_{x}$. If $\tau \in \mathscr{C}_{a, \omega}^{k}$ and $\xi, \eta \in T_{\tau}\left(\mathscr{C}_{a, \omega}^{k}\right)$, then for any $x$ the vectors $\xi(x), \eta(x)$ are tangent vectors of $\mathscr{B}_{x}$ at $\tau(x)$, whose Hermitian product is $m_{x}(\xi(x), \eta(x))_{\tau(x)}$. In fact this is related with $\mathrm{g}_{\tau}$.

Lemma 1.9.

$$
g_{\tau}(\xi, \eta)(x)=m_{x}(\xi(x), \eta(x))_{\tau(x)} \text { for any } \tau \in \mathscr{C}_{a, \omega}^{k}, x \in M .
$$


Proof. We consider any two tangent vectors $\xi$, $\eta$ at $\tau$ as elements of $H^{k}\left(S_{\sigma}^{*} \otimes \bar{S}_{\sigma}\right)$ with respect to the embedding $\mathscr{E}_{a, \omega}^{k} \longrightarrow U(\sigma)$. A differentiation of the map $\kappa: A_{\tau}(\rho) \rightarrow A_{\sigma}(\rho)$ (cf.(1.1)) at $\rho=\tau$ immediately yields as derivative

$$
\begin{aligned}
(D \kappa)(\tau): H^{k}\left(S_{\tau}^{*} \otimes \bar{S}_{\tau}\right) & \longrightarrow H^{k}\left(S_{\sigma}^{*} \otimes \bar{S}_{\sigma}\right) \\
\xi & \longrightarrow\left(\bar{I}_{\tau}-\overline{A_{\tau}(\tau)}\right) \xi\left(I_{\sigma}-A_{\sigma}(\tau)\right)
\end{aligned}
$$

so

$$
\text { (1.6) } \begin{aligned}
g_{c} & (\xi, \eta)(x)=\operatorname{trace}\left(\overline{\mathrm{D} \kappa(\tau)^{-1}(\eta)}(D \kappa)^{-1}(\tau)(\xi)\right) \\
& =\operatorname{trace}\left(\bar{\eta}\left(\bar{I}_{\sigma}-\overline{A_{\sigma}(\tau)}\right)^{-1}\left(\bar{I}_{\tau}-\bar{A}_{\tau}(\sigma)\right)^{-1} \xi\left(I_{\sigma}-A_{\sigma}(\tau)\right)^{-1}\left(I_{\tau}-A_{\tau}(\sigma)\right)^{-1}\right. \\
& =\operatorname{trace}\left(\bar{\eta}\left(\bar{I}_{\sigma}-A_{\sigma}(\tau) \overline{A_{\sigma}(\tau)}\right)^{-1} \xi\left(I_{\sigma}-\overline{A_{\sigma}(\tau)} A_{\sigma}(\tau)\right)^{-1}\right) \\
& =m_{x}(\xi(x), \eta(x))_{\tau(x)} .
\end{aligned}
$$

Proposition 1. 10. The weak Hermitian inner product (1.4) on $\mathbb{T}_{\tau}\left(\mathscr{C}_{a, \omega}^{k}\right)$ depends smoothly on the base point.

Proof. We fix any $\sigma \in \mathscr{C}_{a, \omega}^{k}$. The tangent bundle of $\mathscr{C}_{a, \omega}^{k}$ can be trivialized by the embedding into $U(\sigma)$. Then (1.6) implies that the assignment $(\tau, \xi, \eta) \rightarrow g_{\tau}(\xi, \eta)(x)$ is a $C^{\infty}$ map from

$$
\mathscr{C}_{a, \omega}^{k} \times H^{k}\left(S_{\sigma}^{*} \otimes S_{\sigma}\right) \times H^{k}\left(S_{\sigma}^{*} \otimes S_{\sigma}\right)
$$

to $H^{k}(M)_{c}$. Since $\omega^{m}$ is of class $C^{\infty}$, the assertion holds.

A complex analytic Hilbert manifold with a weak Hermitian metric is called a weak Hermitian symmetric space, if for any of its points $p$ there exists a holomorphic isometric involution with $p$ as an isolated fixed point.

Proposition 1.11. The complex analytic Hilbert manifold $\mathscr{C}_{a, \omega}^{k}$ with the above weak Hermitian metric is a weak Hermitian symmetric space.

Proof. Given an element $\sigma \in \mathscr{C}_{a, \omega}^{k}$, we choose the embedding $\mathscr{C}_{a, \omega}^{k} \rightarrow U(\sigma) \subset H^{k}\left(S^{2}\left(\bar{S}_{\sigma}\right)\right)$. By formulas (1.4) and (1.6) the map $A_{\sigma}(\tau) \rightarrow-A_{\sigma}(\tau)$ is an isometry.

Remark 1.12. More generally, the space of $H^{k}$-sections of any 
$C^{\infty}$ fiber bundle with fiber a Hermitian symmetric space (instead of a Siegel upper half space) and analytic structure group has a natural structure of a weak Hermitian symmetric space.

We can also give a distinguished (strong) Hermitian structure on $\mathscr{C}_{a, \omega}^{k}$. We use Yano's Construction [YA, VI. 6] of a metric connection $D=D_{\sigma}$ on an almost Hermitian manifold with torsion such that the almost complex structure is parallel. It is given with respect to real coordinates by

$$
\Gamma_{j i}^{h}=\left\{\begin{array}{l}
h \\
j i
\end{array}\right\}+\frac{1}{4} N_{i j}^{h},
$$

where $N_{i j}^{h}$ denotes the Nijenhuis torsion tensor. Or, if $X, Y$ are real vector fields, then

$$
D_{X} Y=\frac{1}{2}\left(\nabla_{X} Y-\sigma \nabla_{X}(\sigma Y)\right) .
$$

As $D$ is type-preserving, it induces connections on $\bar{S}_{\sigma} \otimes \bar{S}_{\sigma}$ and $S^{2}\left(\bar{S}_{\sigma}\right)$, which we denote by the same letter. Now on the spaces of $\mathrm{H}^{k_{-}}$ sections of these bundles, Sobolev norms are given by

$$
(\xi, \eta)_{k, \sigma}=\sum_{j=0}^{k} \int_{M} g_{\sigma}\left(D_{\sigma}^{j} \xi, D_{\sigma}^{j} \eta\right)(x) \omega^{m} .
$$

(Here $g_{\sigma}$ is naturally extended to a Hermitian product on spaces of tensors). This assignment turns each tangent space $T_{\sigma}\left(\mathscr{C}_{a, \omega}^{k}\right)$ into a Sobolev space. We will show in the next paragraph that this inner product is invariant under the symplectic diffeomorphism group (as well as the weak product).

\section{The Group of Symplectic $H^{k}$-Diffeomorphisms}

As a non-linear problem, the construction of the group of Sobolev diffeomorphisms on a compact differentiable manifold was treated by Ebin [EB] in the framework of $H^{k}$ sections of a differentiable fiberbundle $F \rightarrow M$ on a compact manifold. If $k>m=\frac{1}{2} \operatorname{dim} M$, then the space $H^{k}(F)$ was given the structure of a $C^{\infty}$ Hilbert manifold that for $\mathrm{k}>m+r$ there is a natural continuous embedding of it into the Banach manifold of $C^{r}$ sections of $F$. 
Mappings of $M$ to itself are treated as sections of the trivial fiber bundle $F=M \times M \rightarrow M$, and the space of $C^{1}$ sections whose inverse exist is an open subset of $C^{1}(F)$. Its intersection with $H^{k}(F)$, $k>m+1$, is called $\mathscr{D}^{k}$, and the group of $C^{\infty}$ diffeomorphisms is denoted by $\mathscr{D}$. Given $s \in H^{k}(F)$ a neighborhood $U$ of $s$, according to Palais' construction, is given as follows: The relative tangent bundle $T(F / M)$ is restricted to the section $s$ and $U$ can be identified with a neighborhood of the zero-section of this bundle. In the case of a trivial bundle $U$ reduces to an open neighborhood of the zero-section of $H^{k}\left(s^{*} T M\right)$, where $s^{*}$ denotes the topological preimage under $s: M \rightarrow M$. In particular, $H^{k}\left(s^{*} T M\right)$ is the tangent space of $\mathscr{D}^{k}$ at $s$.

We will study the action of $\mathscr{D}^{k+1}$ on $\mathscr{C}_{a}^{k}$. First, we list a couple of basic properties of $\mathscr{D}^{k}$ (cf. $[\mathbb{E B}, \mathrm{OM}]$ )。

Proposition 2.1. The set of $H^{k}$ diffeomorphisms $\mathscr{D}^{k}$ on a compact differentiable manifold is a $C^{\infty}$ Hilbert manifold and a topological group for $k>m+1$. The composition of maps induces maps

$$
\mathscr{D}^{k+r} \times \mathscr{D}^{k} \longrightarrow \mathscr{D}^{k} ;(\phi, \varphi) \longrightarrow \phi \circ \varphi
$$

of class $C^{r}$ for $r \geqq 0$. If $\phi \in \mathscr{D}^{k}$, then the right-multiplication $R_{\phi}: \mathscr{D} \rightarrow \mathscr{D}^{k}$ is of class $C^{\infty}$. If $\phi \in \mathscr{D}$, then the left-multiplication $L_{\phi}: \mathscr{D}^{k} \rightarrow \mathscr{D}^{k}$ is of class $C^{\infty}$.

Let $k>m+1$ and $\phi \in \mathscr{D}^{k+1}$ be fixed. Then the continuous map $D \phi: T M \rightarrow T M$ of the tangent bundle over $\phi: M \rightarrow M$ induces a continuous linear map $T_{i d}\left(\mathscr{D}^{k}\right) \rightarrow T_{\phi}\left(\mathscr{D}^{k}\right)$, which is related to the left multiplication by $\phi$. Now for $\sigma \in \mathscr{C}_{a}^{k}$ we have a natural commutative diagram

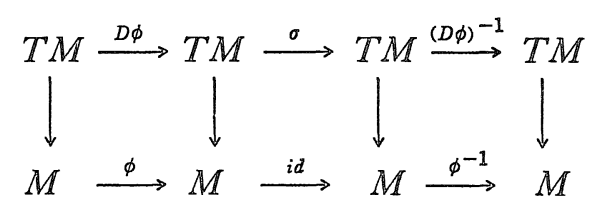

By standard arguments (cf. $[E B, O M]$ ) one can show:

Proposition 2.2. The assignment $\sigma \rightarrow \phi^{*}(\sigma):=(D \phi)^{-1} \sigma(D \phi)$ defines a continuous action

$$
\mu: \mathscr{D}^{k+1} \times \mathscr{C}_{a}^{k} \longrightarrow \mathscr{C}_{a}^{k},
$$


which is of class $C^{\infty}$ in the second variable. If $\sigma \in \mathscr{C}_{a}$ is fixed, then the map $\phi \rightarrow \phi^{*} \sigma$ from $\mathscr{D}^{k+1}$ to $\mathscr{C}_{a}^{k}$ is of class $C^{\infty}$.

Fix any $\phi \in \mathscr{D}$. Then it induces an almost complex map $\left(M, \phi^{*} \sigma\right)$ $\rightarrow(M, \sigma)$ and there is a commutative diagram

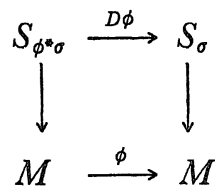

So the derivative of the differentiable map $\mu(\phi,-): \mathscr{C}_{a}^{k} \rightarrow \mathscr{C}_{a}^{k}$ takes the holomorphic tangent space of $\mathscr{C}_{a}^{k}$ to itself;i. e. $\mu(\phi,-)$ is a holomorphic map of $\boldsymbol{C}$-Hilbert manifolds. One can verify immediately that (2.1) implies

$$
A_{\phi^{*} \sigma}\left(\phi^{*} \tau\right)=(D \phi)^{-1} A_{\sigma}(\tau)(D \phi),
$$

which means that $D \mu(\phi,-): T_{\sigma}\left(\mathscr{C}_{a}^{k}\right) \rightarrow T_{\phi^{*} \sigma}\left(\mathscr{C}_{a}^{k}\right)$ maps an element $\xi$ to $(D \phi)^{-1} \xi(D \phi)$. There is also a natural action

$$
\alpha: \mathscr{D}^{k+1} \times H^{k}\left(\Lambda^{2}\left(T^{*}\right)\right) \longrightarrow H^{k}\left(\Lambda^{2}\left(T^{*}\right)\right),
$$

which is similar to $\mu$ with respect to differentiability (cf.2.2). If $\omega \in H^{\infty}\left(\Lambda^{2}\left(T^{*}\right)\right)$ is a fixed symplectic form as in the first paragraph, then $\mathscr{D}_{\omega}^{k+1}$ shall denote its isotropy group, the group of symplectic $\mathrm{H}^{k+1}$ diffeomorphisms. It is well-known (and follows from a consideration of $\alpha(-, \omega)$ that $\mathscr{D}_{\omega}^{k+1}$ is a closed subgroup of $\mathscr{D}^{k+1}$ and a $C^{\infty}$-submanifold. The derivative $D \alpha(-, \omega): H^{k+1}(T) \rightarrow H^{k}\left(\Lambda^{2}\left(T^{*}\right)\right)$ of $\alpha(-, \omega)$ at $\phi=i d$ is given by the the Lie-derivative $X \rightarrow L_{X} \omega$. Its kernel $T_{\omega}^{k+1}$, which is the tangent space of $\mathscr{D}_{\omega}^{k+1}$ at the identity, is called the space of symplectic vector fields. The following is clear:

Proposition 2. 3. The action of $\mathscr{D}^{k+1}$ on $\mathscr{C}_{a}^{k}$ restricts to $\mu_{\omega}: \mathscr{D}_{\omega}^{k+1} \times$ $\mathscr{C}_{a, \omega}^{k} \rightarrow \mathscr{C}_{a, \omega}^{k}$, and any $\phi \in \mathscr{D}_{\omega}^{k+1}$ fixes the closed analytic subspace $\mathscr{C}_{i, \omega}^{k}$ All maps $\mu_{\omega}(\phi,-): \mathscr{C}_{a, \omega}^{k} \rightarrow \mathscr{C}_{a, \omega}^{k}$ are holomorphic. For $\sigma \in \mathscr{C}_{a, \omega}$ the map $\mu_{\omega}(-, \sigma)$ : $\mathscr{D}_{\omega}^{k+1} \rightarrow \mathscr{C}_{a, \omega}^{k}$ is of class $C^{\infty}$. It is also true that the restriction

$$
\mu_{\omega}: \mathscr{D}_{\omega}^{k+1} \times \mathscr{C}_{a, \omega}^{k+r} \longrightarrow \mathscr{C}_{a, \omega}^{k} \text { is of class } C^{r} \text { for } r \geqq 0 \text {. }
$$

Proposition 2.4. The group $\mathscr{D}_{\omega}^{k+1}$ acts on the complex analytic Hilbert manifold $\mathscr{C}_{a, \omega}^{k}$ equipped with both the weak (1.4) and the complete (1.9) Hermitian metric as group of isometries. 
Proof: Let $\phi \in \mathscr{D}_{\omega}^{k+1}$ be fixed. Let $\tau$ be any element of $\mathscr{C}_{a, \omega}^{k}$ and $\tau^{\prime}:=\phi^{*} \tau$. Then by $2.2 \phi:\left(M, \tau^{\prime}\right) \rightarrow(M, \tau)$ is an almost complex map. Let $g=g_{\tau}$ and $g^{\prime}=g_{\tau^{\prime}}$. If $X, Y \in H^{k}(T)$ are real vector fields, then

$$
\begin{aligned}
g\left(\phi_{*} X, \phi_{*} Y\right) & =-\omega\left(\phi_{*} X, \tau \phi_{*} Y\right) \\
& =-\omega\left(\phi_{*} X, \phi_{*} \tau^{\prime} Y\right) \\
& =-\omega\left(X, \tau^{\prime} Y\right) \\
& =g^{\prime}(X, Y) .
\end{aligned}
$$

Thus $\phi$ induces an isometry from $H^{k}\left(S^{2}\left(\bar{S}_{\tau^{\prime}}\right)\right)$ to $H^{k}\left(S^{2}\left(\bar{S}_{\tau}\right)\right)$ provided with the $L^{2}$ inner product.

Now we show that $\phi$ induces an isometry also with respect to the $H^{k}$-complete metric. $\nabla$ and $\nabla^{\prime}$ resp. shall denote the Riemannian connections with respect to $g$ and $g^{\prime}$. We use the connections $D$ and $D^{\prime}$ of Yano's such that $g$ and $\tau\left(g^{\prime}\right.$ and $\tau^{\prime}$ ) are parallel (see formula (1.8)). Then

$$
\begin{aligned}
2 \phi_{*}\left(\mathrm{D}_{X}^{\prime} Y\right) & =\phi_{*} \nabla_{X}^{\prime} Y-\phi_{*} \tau^{\prime} \nabla_{X}^{\prime}\left(\tau^{\prime} Y\right) \\
& =\nabla_{\phi_{*} X}\left(\phi_{*} Y\right)-\phi_{*} \tau^{\prime}\left(\phi_{*}\right)^{-1}\left(\left(\phi_{*} \nabla_{x}^{\prime} \phi_{*}^{-1}\right)\left(\phi_{*} Y\right)\right) \\
& =\nabla_{\phi_{*} X}\left(\phi_{*} Y\right)-\tau \nabla_{\phi_{*} X}\left(\tau \phi_{*} Y\right)=2 D_{\phi_{*} X}\left(\phi_{*} Y\right) .
\end{aligned}
$$

This formula is still true if we replace $Y$ by any symmetric tensors. If $\left\{X_{i}\right\}$ is a set of $g^{\prime}$-orthonormal vector fields, then

$$
\sum_{i} g\left(D_{\phi_{*} X_{i}} \phi_{*} Y, D_{\phi_{*} X_{i}} \phi_{*} Z\right)=\sum_{i} g^{\prime}\left(D_{X_{i}} Y, D_{X_{i}}^{\prime} Z\right) \text {. }
$$

From this and analogous equalities one can deduce easily the desired assertion.

\section{The Space of Isomorphic Almost $\mathbb{K}$ ähler Structures and the Action of the Group of Symplectic Diffeomorphisms}

Let $\sigma$ and $\tau$ be Kähler structures on $(M, \omega)$ of class $H^{k}$. Then two Kähler manifolds $(M, \sigma, \omega)$ and $(M, \tau, \omega)$ are isomorphic, if there exists a biholomorphic map $\varphi:(M, \sigma) \rightarrow(M, \tau)$ such that $\varphi^{*} \omega=\omega$. In this section we show first that such a $\varphi$ is necessarily of class $H^{k+1}$. This means that we can identify the set of isomorphism classes of such Kähler manifolds with the quotient space $\mathscr{M}_{\omega}^{k}=\mathscr{C}_{i, \omega}^{k} / \mathscr{D}_{\omega}^{k+1}$. We show furthermore that the action of $\mathscr{D}_{\omega}^{k+1}$ on $\mathscr{C}_{i, \omega}^{k}$ is proper.

Theorem 3.1. Let $k>2 m+3$ and $\rho, \tau \in \mathscr{C}_{a, \omega}^{k}$. Let $g_{\rho}$ and $g_{\tau}$ be the 
respective underlying Riemannian metrics (which are in particular of class $\left.C^{m+3}\right)$. Then any symplectic $C^{1}$ isometry $\phi:\left(M, g_{\rho}\right) \rightarrow\left(M, g_{\tau}\right)$ is necessarily of class $H^{k+1}$.

Corollary 3.2. The moduli spaces $\mathscr{M}_{\omega}^{k}$ and $\mathscr{M}_{a, \omega}^{k}$ of isomorphism classes of (almost) Kähler structures of class $H^{k}$ on the symplectic manifold $(M, \omega)$ are exactly the quotients $\mathscr{C}_{i, \omega}^{k} / \mathscr{D}_{\omega}^{k+1}$ and $\mathscr{C}_{a, \omega}^{k} / \mathscr{D}_{\omega}^{k+1}$.

Theorem 3. 3. The action $\mu_{\omega}: \mathscr{D}_{\omega}^{k+1} \times \mathscr{C}_{a, \omega}^{k} \rightarrow \mathscr{C}_{a, \omega}^{k}$ is proper, provided $k>2 m+3$ 。

Corollary 3.4. The spaces $\mathscr{M}_{\omega}^{k}$ and $\mathscr{M}_{a, \omega}^{k}$ equipped with the quotient topology are Hausdorff and the embeddings $\mathscr{C}_{a, \omega}^{k+1} \rightarrow \mathscr{C}_{a, \omega}^{k}, \mathscr{C}_{i, \omega}^{k+1} \rightarrow \mathscr{C}_{i, \omega}^{k}$ yield embeddings $\mathscr{M}_{a, \omega}^{k+1} \rightarrow \mathscr{M}_{a, \omega}^{k}$ and $\mathscr{M}_{\omega}^{k+1} \rightarrow \mathscr{M}_{\omega}^{k}$.

Corollary 3.5. (i) All $\mathscr{D}_{\omega}^{k+1}$-orbits are closed in $\mathscr{C}_{a, \omega}^{k}$.

(ii) If $\tau \in \mathscr{C}_{a, \omega}^{k}$, then the isotropy group $\mathscr{D}_{\omega, \tau}^{k+1}$ is compact.

Lemma 3.6. Let $g_{j}, j \in N$ and $g_{0}$ be Riemannian metrics on a manifold $M$ and $d_{j}, d_{0}$ the corresponding distance functions. If $g_{j}$ converge to $g_{0}$ in a locally uniform way, then $d_{j} \rightarrow d_{0}$ in a locally uniform way.

Proof. Ghoose a compact coordinate neighborhood $U$ in $M$ and denote by $l_{j}(\gamma)$ the $g_{j}$-length of an arbitrary $C^{1}$-path $\gamma:(0,1) \rightarrow$ $U, j \geqq 0$. From the assumption we get for any $1>\varepsilon>0$ a $j_{0}$ such that $\left|l_{j}(\gamma)-l_{0}(\gamma)\right|<\varepsilon l_{0}(\gamma)$ for $j \geqq j_{0}$. This implies $d_{j}(x, y) \leqq(1+\varepsilon) \circ d_{0}(x, y)$ and $d_{0}(x, y) \leqq \frac{1}{1-\varepsilon} \circ d_{j}(x, y)$ for all $x, y \in U$ and $j \geqq j_{0}$ 。

Lemma 3.7. Let $M, \tilde{M}$ be connected locally compact metric spaces with metrics $d_{n}, \tilde{d}_{n}$ converging in a locally uniform way to metrics $d_{0}$ and $\tilde{d}_{0}$ respectively. If $\Phi_{j}:\left(M, d_{j}\right) \rightarrow\left(\tilde{M}, \tilde{d}_{j}\right)$ are isometries, then a subsequence $\Phi_{j(k)}$ converges in a locally uniform way to an isometry $\Phi_{0}:\left(M, d_{0}\right) \rightarrow\left(\tilde{M}, \tilde{d_{0}}\right)$.

The proof of the classical v. Dantzig-v. d. Waerden theorem gives the above statement immediately (cf. $[K-N, \mathbb{I} .4]$ ).

Let $M$ be a $C^{\infty}$ manifold. Let $r>0$ and $g, h$ be Riemannian metrics of class $C^{r+1}$. Then any isometry $\phi: M \rightarrow M$ of the underlying metric 
spaces is by the Meyers-Steenrod theorem of class $C^{r}$. Moreover we have the following assertion:

Lemma 3.8. Let $g_{j}$ and $h_{j}, j \in \mathbb{N}$, be Riemannian metrics of class $C^{r+1}$ on $M$, which converge uniformly on compact sets together with their derivatives up to order $r+1$, i.e. in the $C^{r+1}$-topology, to Riemannian metrics $g_{0}$ and $h_{0}$ respectively. Let $\Phi_{j}: M \rightarrow M$ be isometries of the metric spaces induced by $g_{j}$ and $h_{j}$ respectively. Then a subsequence $\Phi_{j(k)}$ converges in the $C^{r}$-topology to a $C^{r}$-isometry $\Phi_{0}:\left(M, g_{0}\right) \rightarrow\left(M, h_{0}\right)$.

Proof: By 3.6 and 3.7 a subsequence $\Phi_{j(k)}$ converges in the $C^{0}$-topology to some $C^{0}$-isometry $\Phi_{0}: M \rightarrow M$ of the metric spaces induced by $g_{0}$ and $h_{0}$ respectively. Let $L(M)$ be the principal bundle of linear frames on $M$. The metrics $g_{j}$ and $h_{j}$ resp. induce Riemannian metrics $\tilde{g}_{j}$ and $\tilde{h}_{j}$ of class $C^{r}$ on $L(M)$ which converge to $\tilde{g}_{0}$ and $\tilde{h}_{0}$ resp. in the $C^{r}$-topology。 (The assignment $g \rightarrow \tilde{g}$ is as follows: For the vertical direction embed $L(M)$ into the Riemannian vector bundle $\operatorname{End}(T M)$ and induce the flat metric of each fiber of $\operatorname{End}(T M)$ on each fiber of $L(M)$. For the horizontal direction use the Riemannian connection.) Now the $\Phi_{j *}: L(M) \rightarrow L(M)$ are isometries of the metric spaces induced by $\tilde{g}_{j}$ and $\tilde{h}_{j}$ respectively. Again by 3.6 and 3.7 a subsequence $\Phi_{\tilde{j}(k) *}$ converges in the $C^{0}$-topology to $\Phi_{0^{*}}$, which means that $\Phi_{\tilde{j}(k)}$ converges to $\Phi_{0}$ in the $C^{1}$-topology. The rest follows by a repeated application of this argument.

Proof of 3.1. By the theorem of Meyers-Steenrod any isometry $\phi$ is of class $\mathbb{C}^{m+2}$. The claim is of local nature. For simplicity we assume the existence of a reference Kähler structure $\sigma$ which induces the underlying differentiable structure. (This is the only case which is of interest to us.) Let $V$ and $W$ be coordinate neighborhoods with local holomorphic coordinates $\left(z_{1}, \ldots, z_{m}\right)$ and $\left(w_{1}, \ldots, w_{m}\right)$ resp. on $(M, \sigma)$ such that $\phi(W) \subset V$. Let $S_{\tau}$ and $S_{\rho}$ resp. be the images of $I_{\sigma}-A$ and $I_{\sigma}-B$ resp. for $A, B \in H^{k}\left(S_{\sigma}^{*} \otimes \bar{S}_{\sigma}\right)$. Then by an elementary calculation, we see that $\phi$ is a solution of the following equations:

$$
\begin{gathered}
\frac{\partial \phi^{i}(w)}{\partial w^{\tilde{s}}}+A^{i}{ }_{l}(\phi(w)) \cdot \frac{\partial \phi^{i}(w)}{\partial w^{\tilde{s}}}-\frac{\partial \phi^{i}(w)}{\partial w^{k}} \cdot B_{s}^{k}(w) \\
-A^{i}{ }_{l}(\phi(w)) \cdot \frac{\partial \phi^{i}(w)}{\partial w^{k}} B_{\bar{s}}^{k}(w)=0
\end{gathered}
$$


where $\phi^{i}$ are the component functions of $\phi$. Then $\left(\phi^{i}\right)$ is a solution of the linear first order system of differential equations

$$
\begin{aligned}
g^{r s}(w) \cdot \frac{\partial u^{i}(w)}{\partial w^{5}} & +g^{r s}(w) \cdot A_{i}^{i}(\phi(w)) \cdot \frac{\partial u^{i}(w)}{\partial w^{5}}-\frac{\partial u^{i}(w)}{\partial w^{k}} \cdot B^{k r}(w) \\
& -A_{i}^{i}(\phi(w)) \cdot \frac{\partial u^{i}}{\partial w^{k}} \cdot B^{k r}(w)=0 .
\end{aligned}
$$

The application of $\sum \frac{\partial}{\partial w^{r}}$ yields a second order system of differential equations, whose main part is

$$
\begin{aligned}
g^{s r}(w) \frac{\partial^{2} u^{i}}{\partial w^{5} \partial w^{r}} & +A_{i}^{i}(\phi(w)) \cdot g^{s r}(w) \cdot \frac{\partial^{2} u^{i}}{\partial w^{5} \partial w^{r}}-B^{k r}(w) \cdot \frac{\partial^{2} u^{i}}{\partial w^{k} \partial w^{r}} \\
& -B^{k r}(w) \cdot A_{i}^{i}(\phi(w)) \cdot \frac{\partial^{2} u^{i}}{\partial w^{k} \partial w^{r}} .
\end{aligned}
$$

The characteristic matrix is the following product for $\left(\xi_{1}, \ldots, \xi_{m}\right) \in \mathbb{C}^{m}$.

$$
\left[\begin{array}{cc}
\left(g^{j k} \bar{\xi}_{j} \xi_{k}-B^{k r} \xi_{k} \xi_{r}\right) I, & 0 \\
0 & ,\left(g^{j k} \bar{\xi}_{j} \xi_{k}-\bar{B}^{k r} \xi_{k} \xi_{r}\right) I
\end{array}\right] \cdot\left[\begin{array}{cc}
I & A^{i}{ }_{i} \\
\bar{A}^{i} & I
\end{array}\right] .
$$

Its determinant is $\left|\mathrm{g}^{j k} \bar{\xi}_{j} \xi_{k}-B^{k r} \xi_{k} \xi_{r}\right|^{2 m} \cdot \operatorname{det}\left(\delta_{k}^{i}-A_{l}^{i} \bar{A}^{i}{ }_{k}\right)$, which is positive whenever $\xi \neq 0$. From this one can see that the above system is elliptic. We write (3.1) in the following form:

$$
a_{\beta}^{\alpha i j}(w, \phi(w)) \cdot \frac{\partial \phi^{\beta}}{\partial w^{i}}=0,
$$

where $a_{\beta}^{\alpha i j}$ are $H^{k}$ functions on $V \times W$ for $i, j, \beta=1, \ldots, m, \overline{1}, \ldots, \bar{m}$ and $\alpha=1, \ldots, m$. We consider the following second order system which is solved by $u=\phi$ :

$$
D_{j}\left(a_{\beta}^{\alpha i j}(w, \phi(w)) \circ D_{i} u^{\beta}\right)=0
$$

Now $\phi$ is already known to be of class $H^{m+2}$. By the $H^{l}$-composition lemma (cf. $[\mathrm{EB}, 3.1]$ ) all $a_{\beta}^{\alpha i j}\left(w, \phi(w)\right.$ ) are of class $H^{m+2}$. (Observe that one needs for this the particular form of the coefficients.) Hence we can use the regularity theorem below for a bootstrap argument to show that $\phi$ is of class $H^{k+1}$.

Theorem 3.9. Let $\Omega^{\prime} \Subset \Omega \Subset \boldsymbol{R}^{n}$ be domains with smooth boundaries. Consider an elliptic system of second order real linear differential equations

$$
L w=D_{i}\left(a_{\lambda}^{\kappa i j} D_{j} w^{\lambda}\right)=f^{\chi}
$$

on $\Omega$ such that $a_{\lambda}^{\kappa i j} \in H^{k+1}(\Omega), f \in H^{k}(\Omega), i, j=1, \ldots, n, \chi, \lambda=1, \ldots, l$ 
and some $k>\frac{n}{2}+1$. Then any weak solution $w=\left(w^{k}\right)$ which is in $H^{1}(\Omega)$ is actually in $H^{k+2}\left(\Omega^{\prime}\right)$, and

$$
\|w\|_{H^{k+2}\left(\Omega^{\prime}\right)} \leqq C \cdot\left(\|w\|_{H^{1}(\Omega)}+\|f\|_{H^{k}(\Omega)}\right)
$$

with $C>0$ only depending continuously on the $H^{k+1}$-norms of the coefficients of $L$ and on the geometry of $\Omega^{\prime} \Subset \Omega \Subset \mathbb{R}^{n}$.

This is an analogy of the main theorem of $[D-N]$ and [G-T, $8.8,8.10]$ and can be proved in parallel way.

Remark 3.10. One can also show the following:

(i) Let $g, g^{\prime}$ be any Riemannian metrics of class $H^{k}$. Then any isometry $\phi:(M, g) \rightarrow\left(M, g^{\prime}\right)$ is necessarily of class $H^{k+1}$ 。

(ii) The action of $\mathscr{D}^{k+1}$ on the space of $H^{k}$ Riemannian metrics is proper.

Proof of 3.3. We have to show: Given $\tau_{j} \in \mathscr{C}_{a, \omega}^{k}$ and $\phi_{j} \in \mathscr{D}_{\omega}^{k+1}$ such that $\rho_{j}=\mu_{\omega}\left(\phi_{j}, \tau_{j}\right)$ and $\tau_{j}$ converge to some $\rho_{0}, \tau_{0}$ in $\mathscr{C}_{a, \omega}^{k}$, there exists a converging subsequence $\phi_{j(c)} \rightarrow \phi_{0}$ in $\mathscr{D}_{\omega}^{k+1}$ (and $\left.\mu_{\omega}\left(\phi_{0}, \tau_{0}\right)=\rho_{0}\right)$. We consider $\phi_{j}:\left(M, g_{\rho_{j}}\right) \rightarrow\left(M, g_{\tau_{j}}\right)$ as a sequence of isometries of Riemannian manifolds. We set $g_{j}=g_{\rho_{j}}$ and $h_{j}=h_{\tau_{j}}$. By 3.8 there exists a subsequence (say $\left\{\phi_{l}\right\}$ itself) which converges to some $C^{m+2}$ symplectic isometry $\phi_{0}$ in the $C^{m+2}$-topology. All $\phi_{c}$ are solutions of

$$
a_{\beta,(l)}^{a i j}\left(w, \phi_{\iota}(w)\right) \circ D_{j} u^{\beta}=0
$$

where $a_{\beta,(l)}^{\alpha i j}(w, z) \rightarrow a_{\beta,(0)}^{\alpha i j}(w, z)$ in the $H^{k}$-topology。 Now we apply 3.1 and the estimates of 3.9. By the composition lemma

$$
a_{\beta,(\iota)}^{\alpha i j}\left(w, \phi_{\iota}(w)\right) \longrightarrow a_{\beta,(0)}^{\alpha i j}\left(w, \phi_{0}(w)\right)
$$

in the $H^{m+2}$-topology. As the constant $C$ in $(3.5)$ depends continuously on the norms of the coefficients, we can see that $\phi_{l} \rightarrow \phi_{0}$ in the $H^{m+2}$ topology. (Consider the equation

$$
a_{\beta,(\iota)}^{\alpha i j}\left(w, \phi_{\iota}\right) \cdot D_{j} u^{\beta}=\left(a_{\beta,(\kappa)}^{\alpha i j}\left(w, \phi_{\kappa}\right)-a_{\beta,(\iota)}^{\alpha i j}\left(w, \phi_{\iota}\right)\right) D_{j} \circ \phi_{\iota}^{\beta}
$$

which is solved by $\phi_{c}-\phi_{\kappa}$ ) 


\section{An Infinitesimal Slice Theorem-Orthogonal Decomposition of the Space of Symmetric $(0,2)$-Tensors}

The first step for the local description of the moduli spaces $\mathscr{M}_{\omega}^{k}$ is to study the infinitesimal action of the group of symplectic diffeomorphisms on the spaces of Kähler and almost Kähler structures. We fix a Kähler structure $\sigma \in \mathscr{C}_{i, \omega}$ and denote by $g$ the metric tensor induces by $\sigma$ and $\omega$.

Lemma 4. 1. Let $X=\left(X^{\alpha} \frac{\partial}{\partial z^{\alpha}}+X^{\beta} \frac{\partial}{\partial z^{\beta}}\right) \in H^{k+1}(T), m+1<k \leqq \infty$ be a real vector field on $(M, \sigma)$. Then $X$ is a symplectic vector field, if and only if

$$
\begin{aligned}
& X_{\beta ; \bar{\delta}}=X_{\delta ; \beta} \\
& X_{\alpha ; \beta}+X_{\tilde{\beta} ; \alpha}=0
\end{aligned}
$$

These conditions are equivalent to

$$
d^{c} \xi=0, \text { where } \xi=X_{\alpha} d z^{\alpha}+X_{\beta} d z^{\beta} \text { and } d^{c}=\sqrt{-1}(\partial-\tilde{\partial}) .
$$

An immediate consequence is:

Lemma 4.2. The symplectic vector fields $X$ of class $H^{k+1}$ on $M$ correspond exactly to real 1 -forms $\xi$ with

$$
X_{\alpha}=\sqrt{-1} f_{; \alpha}+Y_{\alpha}, \quad X_{\beta}=-\sqrt{-1} f_{; \beta}+\overline{Y_{\beta}}
$$

with a real function $f$ of class $H^{k+2}$ on $M$ and a holomorphic 1-form $\eta=Y_{\alpha} d z^{\alpha} \in \Omega^{1}(M, \sigma)$ on $(M, \sigma)$ i.e.

$$
\xi=d^{c} f+\eta+\bar{\eta} \text {. }
$$

From (4.5) one sees that

$$
T_{\omega}^{k+1}=d^{c}\left(H^{k+2}(\boldsymbol{R})\right) \oplus\left(\operatorname{Re} \Omega^{1}(M, \sigma)\right)
$$

is an orthogonal decomposition. We denote by $S^{2}=S_{\sigma}^{2}=S^{2}\left(S_{\sigma}^{*}\right)$ the bundle of (complex) symmetric covariant $(0,2)$-tensors on $(M, \sigma)$ interpreted as an $\boldsymbol{R}$-vector bundle.

Then we consider the following differential operator

$$
\theta: C^{\infty}(\boldsymbol{R}) \longrightarrow C^{\infty}\left(S^{2}\right), \quad \theta(f)=\sqrt{-1} f_{; \bar{\beta} \bar{\delta}}
$$


The symbol of $\theta$ is clearly injective and its formal adjoint

$$
\begin{aligned}
& \theta^{*}: C^{\infty}\left(S^{2}\right) \longrightarrow C^{\infty}(\mathbb{R}) \text { is } \\
& \theta^{*}\left(Y_{\overline{\beta \delta}}\right)=\operatorname{Im}\left(Y_{\overline{\beta \delta} ; \alpha \gamma} g^{\alpha \beta} g^{\gamma \delta}\right) .
\end{aligned}
$$

Both $\theta$ and $\theta^{*}$ are of degree two and map $H^{k+2}$-sections to $H^{k}$-sections. Standard arguments (cf. [BE-EB, Sect. 4]) yield:

Theorem 4. 3. There is a decomposition

$$
\begin{aligned}
H^{k}\left(S^{2}\right) & =\operatorname{Im}\left(\theta: H^{k+2}(\mathbb{R}) \longrightarrow H^{k}\left(S^{2}\right)\right) \\
& \oplus \operatorname{ker}\left(\theta^{*}: H^{k}\left(S^{2}\right) \longrightarrow H^{k-2}(\mathbb{R})\right), \quad k \leqq \infty
\end{aligned}
$$

into the direct sum of closed linear subspaces, which are orthogonal with respect to the $L_{2}$ inner product (, ) o and

$$
H^{k+1}\left(S^{2}\right) \cap \theta\left(H^{k+2}(\mathbb{R})\right)=\theta\left(H^{k+3}(\mathbb{R})\right) \text {. }
$$

We denote by $V$ the finite dimensional image of $\Omega^{1}(M, \sigma)$ in $C^{\infty}\left(S^{2}\right)$ under the map $\eta=Y_{\alpha} d z^{\alpha} \mapsto \bar{Y}_{\beta ; \delta}$ (Observe that $\left.d \eta=0\right)$. The image $\theta\left(H^{k+2}(\mathbb{R})\right)+V$ is closed in $H^{k}\left(S^{2}\right)$ for all $k \leqq \infty$, and if $V^{\perp}$ is the orthogonal complement of $V$ in $H^{0}\left(S^{2}\right)$ with respect to the $L_{2}$ inner product, then $\left(V^{\perp} \cap H^{k}\left(S^{2}\right)\right) \oplus V=H^{k}\left(S^{2}\right)$ in an orthogonal decomposition with respect to $(,)_{0}$. This proves:

Remark 4.4. $H^{k}\left(S^{2}\right)=\left[\theta\left(H^{k+2}(\mathbb{R})\right)+V\right] \oplus\left[\theta_{k}^{*-1}(0) \cap V^{\perp}\right], \quad$ where $\theta_{k}^{*}: H^{k}\left(S^{2}\right) \rightarrow H^{k-2}(\mathbb{R})$ is the formal adjoint of $\theta$. Furthermore

$$
H^{k+1}\left(S^{2}\right) \cap\left(\theta\left(H^{k+2}(\mathbb{R})\right)+V\right)=\theta\left(H^{k+3}(\mathbb{R})\right)+V
$$

and

$$
H^{k+1}\left(S^{2}\right) \cap\left(\theta_{k}^{*-1}(0) \cap V^{\perp}\right)=\theta_{k+1}^{*-1}(0) \cap V^{\perp}
$$

Remark 4.5. If all holomorphic vector fields on $(M, \sigma)$ vanish identically, then $V \cap \theta\left(H^{k+2}(\mathbb{R})\right)=(0)$.

The above theorem is related to the infinisitesimal action of $\mathscr{D}_{\omega}^{k+1}$ on $\mathscr{C}_{a, \omega}^{k}$ :

Proposition 4.6. Let $\sigma \in \mathscr{C}_{i, \omega}^{\infty}$. Then the derivative of $\mu_{\omega}(-, \sigma)$ : $\mathscr{D}_{\omega}^{k+1} \rightarrow \mathscr{C}_{a, \omega}^{k}$ at the identity, $D \mu_{\omega}(-, \sigma)_{i d}: T^{k+1} \rightarrow H^{k}\left(S_{\sigma}^{2}\right)$, is given by 


$$
X=X^{\alpha} \frac{\partial}{\partial z^{\alpha}}+X^{\beta} \frac{\partial}{\partial z^{\beta}} \rightarrow X_{\beta ; \delta} .
$$

Its image is the (closed) subspace $\theta\left(H^{k+2}(\boldsymbol{R})\right)+V$.

Let $H_{0}^{k+2}(M, \boldsymbol{R})=\left\{f \in H^{k+2}(M, \boldsymbol{R}) ; \int f \omega^{m}=0\right\}$, which is the orthogonal complement of the constants with respect to the $L_{2}$ inner product in $H^{k+2}(M, \boldsymbol{R})$. Then in the above arguments we may replace $d^{c}$ by its injective restriction to $H_{0}^{k+2}(M, R)$ and $\theta$ by its restriction

$$
\theta: H_{0}^{k+2}(M, \boldsymbol{R}) \longrightarrow H^{k}\left(M, S^{2}\right) \text {. }
$$

\section{The Slice Theorem for the Action of the Group of Symplectic Diffeomorphisms on the Space of Almost Complex Structures}

Roughly speaking, a slice for the action of $\mathscr{D}_{\omega}^{k+1}$ on $\mathscr{C}_{a, \omega}^{k}$ and $\mathscr{C}_{i, \omega}^{k}$ at a point $\sigma \in \mathscr{C}_{i, \omega}^{k}$ is a subspace complementary to the orbit and invariant under the isotropy group of $\sigma$. As $\mu_{\omega}$ is just of class $C^{0}$, we shall have to restrict ourselves to structures $\sigma$ of class $C^{\infty}$. In this section we always assume $k>2 m+3$.

Let $\sigma \in \mathscr{C}_{i, \omega}$ be a fixed Kähler structure. Then $\mu_{\omega}(-, \sigma): \mathscr{D}_{\omega}^{k+1} \rightarrow \mathscr{C}_{a, \omega}^{k}$ is of class $C^{\infty}$, its image, the orbit $O_{\sigma}^{k}$, is closed in $\mathscr{C}_{a, \sigma}^{k}$ by 3.5 , and its isotropy group $\mathscr{D}_{\sigma}$ is compact and by 3.1 contained in $\mathscr{D}_{\omega}$. Furthermore, as $\sigma$ is integrable, it consists of biholomorphic automorphisms of $(M, \sigma)$.

Lemma 5.1. The space $\mathscr{D}_{\omega}^{k+1} / \mathscr{D}_{\sigma}$ of left cosets $\phi \cdot \mathscr{D}_{\sigma}$ carries a natural structure of a $C^{\infty}$-Hilbert manifold, and the map $\mu_{\omega}(-, \sigma)$ : $\mathscr{D}_{\omega}^{k+1} \rightarrow O_{\sigma}^{k} \subset \mathscr{C}_{a, \omega}^{k}$ induces a diffeomorphism from $\mathscr{D}_{\omega}^{k+1} / \mathscr{D}_{\sigma}$ onto $O_{\sigma}^{k}$. All of these maps are compatible with the Sobolev filtration.

Proof. As $\mathscr{D}_{\sigma}$ is compact, the quotient $\mathscr{D}_{\omega}^{k+1} / \mathscr{D}_{\sigma}$ is Hausdorff.

As $\mathscr{D}_{\sigma} \subset \mathscr{D}_{\omega}^{\infty}$, the multiplication $\mathscr{D}_{\omega}^{k+1} \times \mathscr{D}_{\sigma} \rightarrow \mathscr{D}_{\omega}^{k+1}$ is of class $C^{\infty}$, and the tangent space of $\mathscr{D}_{\sigma}$ is closed in $\mathscr{D}_{\omega}^{k+1}$. The derivative of the above map is just the addition of tangent vectors. Thus we can find through every given point $\phi$ (locally closed) submanifolds $A^{k+1} \subset \mathscr{D}_{\omega}^{k+1}$ such that the multiplication restricted to $A^{k+1} \times \mathscr{D}_{\sigma}$ is a diffeomorphism 
on to an open subset of $\mathscr{D}_{\omega}^{k+1}$. By the canonical projection $A^{k+1}$ is mapped onto some open set $V^{k+1} \subset \mathscr{D}_{\omega}^{k+1} / \mathscr{D}_{\sigma}$ homeomorphically, which can be taken as a coordinate neighborhood. Transition functions can be induced by the left-multiplication of elements of $\mathscr{D}_{\omega}$. If $\psi \in \mathscr{D}_{\omega}$ then we can achieve $A^{k+1} \cap \mathscr{D}_{\omega}^{k+2}=A^{k+2}$ for any $k>2 m+3$. Now $\mu_{\omega}(-, \sigma)$ can be factored by a $C^{\infty}$ map $\mathscr{D}_{\omega}^{k+1} / \mathscr{D}_{\sigma} \rightarrow O_{\sigma}^{k}$ and from 4.6 its derivative is a closed embedding.

We consider the closed submanifold $O_{\sigma}^{k} \subset \mathscr{C}_{a, \omega}^{k}$. Its normal bundle $\nu^{k}$ is by definition the orthogonal complement of the tangent bundle $T\left(O_{\sigma}^{k}\right)$ in $T\left(\mathscr{C}_{a, \omega}^{k}\right) \mid O_{\sigma}^{k}$ with respect to the $L_{2}$ inner product on the fibers of $T\left(\mathscr{C}_{a, \omega}^{k}\right) \mid O_{\sigma}^{k}$. The bundle $\nu^{k}$ is homogeneous under the action of $\mathscr{D}_{\omega}^{k+1}$ in the strong sense that the action on $O_{\sigma}^{k}$ lifts to the total space of the bundle. If $\tau \in \mathscr{C}_{a, \omega}^{k}$ and $\phi \in \mathscr{D}_{\omega}^{k+1}$, then we write $\phi^{*} \tau:=$ $\mu_{\omega}(\phi, \tau)$.

Proposition 5.2. The normal bundle $\nu^{k}$ is a $C^{\infty}$ subbundle of $T\left(\mathscr{C}_{a, \omega}^{k}\right) \mid O_{\sigma}^{k}$

Proof. Let $\mathscr{D}_{\omega}^{k+1} \times T_{\sigma}\left(\mathscr{C}_{a, \omega}^{k}\right) \rightarrow T\left(\mathscr{C}_{a, \omega}^{k}\right) \mid O_{\sigma}^{k}$ be the natural continuous action where $T_{\sigma}\left(\mathscr{C}_{a, \omega}^{k}\right)$ is the tangent space at $\sigma$. Let $\tilde{\nu}^{k} \subset T\left(\mathscr{C}_{a, \omega}^{k}\right) \mid O_{\sigma}^{k}$ be the image of $\mathscr{D}_{\omega}^{k+1} \times \theta^{*-1}(0)$. Then exactly as in ([EB], proof of 7. 1) one can show that $\tilde{\nu}^{k}$ is a smooth subbundle. Now $\nu^{k}$ is just the image of $\mathscr{D}_{\omega}^{k+1} \times\left(T\left(O_{o}^{k}\right)^{\perp}\right)$. By 4.3 we have the orthogonal decomposition $C^{\infty}\left(S^{2}\right)=\operatorname{Im} \theta \oplus \operatorname{Ker} \theta^{*}$. Let $V^{\prime}$ be the image of $V$ in $\operatorname{Ker} \theta^{*}$ by the resulting projection. Then we have the orthogonal decomposition $\tilde{\nu}^{k}=\operatorname{Im} \theta_{k} \oplus V^{\prime}$ with $V^{\prime} \subset \operatorname{Ker} \theta_{k}^{*}$. Let $\mathscr{V}^{\prime}$ be the image of $\mathscr{D}_{\omega}^{k+1} \times V^{\prime} \rightarrow T_{\sigma}\left(\mathscr{C}_{a, \omega}^{k}\right) \mid O_{\sigma}^{k}$ by the above action. Then $\mathscr{V}^{\prime}$ is clearly a $C^{\infty}$-subbundle of finite rank of $\tilde{\nu}^{k}$. Let $\lambda: \tilde{\nu}^{k} \rightarrow \mathscr{V}^{\prime}$ be the orthogonal projection, which is a $C^{\infty}$ homomorphism. Moreover it is a submersion because the restriction of $\lambda$ to $\mathscr{V}^{\prime}$ is the identity. Hence $\nu^{k}=\operatorname{Ker} \lambda$ is a $C^{\infty}$ subbundle.

Corollary 5. 3. There exists a $\mathscr{D}_{\omega}^{k+1}$-invariant neighborhood $W^{k}$ of the zero-section of $\nu^{k}$ which is mapped diffeomorphicallv by the exponential map onto a neighborhood $N^{k}$ of $O_{\omega}^{k}$ in $\mathscr{C}_{a, \omega}^{k}$. The map is $\mathscr{D}_{\omega}^{k+1}$-equivariant.

Remark 5.4. We can choose $W^{k_{0}}$ and $N^{k_{0}}$ for some $k_{0} \geqq 2 m+3$ such that $W^{k}:=W^{k_{0}} \cap \nu^{k}$ and $N^{k}:=N^{k_{0}} \cap \mathscr{C}_{a, \omega}^{k}$ for $k>k_{0}$ have the above 
property. This follows from our construction and [KO] (in particular lemma 2.8).

Definition 5. 5. Let $\sigma \in \mathscr{C}_{i, \omega}$ and $k>2 m+3$. Then a slice $\mathscr{S}^{k}$ through $\sigma$ for the action of $\mathscr{D}_{\omega}^{k+1}$ on $\mathscr{C}_{a, \omega}^{k}$ is a locally closed $C^{\infty}$-submanifold of $\mathscr{C}_{a, \omega}^{k}$ with $\sigma \in \mathscr{S}^{k}$, which satisfies the following conditions:

(i) $\mathscr{S}^{k}$ is invariant under the action of the isotropy group $\mathscr{D}_{\sigma} \subset \mathscr{D}_{\omega} \subset \mathscr{D}_{\omega}^{k+1}$.

(ii) If $\phi \in \mathscr{D}_{\omega}^{k+1}$ with $\mu_{\omega}\left(\phi, \mathscr{S}^{k}\right) \cap \mathscr{S}^{k} \neq \emptyset$ then $\phi \in \mathscr{D}_{0}$.

(iii) There exist a neighborhood $V^{k+1} \subset \mathscr{D}_{\omega}^{k+1} / \mathscr{D}_{\sigma}$ of the residue class of the identity and a section $\beta: V^{k+1} \rightarrow \mathscr{D}_{\omega}^{k+1}$ of $\mathscr{D}_{\omega}^{k+1} \rightarrow \mathscr{D}_{\omega}^{k+1} / \mathscr{D}_{\sigma}$ such that $\mu_{\omega}(\beta \times$ id $): V^{k+1} \times \mathscr{S}^{k} \rightarrow \mathscr{C}_{a, \omega}^{k}$ is a homeomorphism onto a neighborhood of $\sigma$.

Theorem 5.6. Let $\sigma \in \mathscr{C}_{i, \omega}$ and $k>2 m+3$. Then there exists always a slice $\mathscr{S}^{k}:=\mathscr{S}_{\sigma}^{k}$ through $\sigma$ for the action of $\mathscr{D}_{\omega}^{k+1}$ on $\mathscr{C}_{a, \omega}^{k}$. The set $\mathscr{S}_{i}^{k}:=\mathscr{S}_{i, \phi}^{k}:=\mathscr{S}^{k} \cap \mathscr{C}_{i, \omega}^{k}$ is closed in $\mathscr{S}^{k}$ and the statements (i) to (iii) with $\mathscr{S}^{k}$ and $\mathscr{C}_{a, \omega}^{k}$ replaced by $\mathscr{S}_{i}^{k}$ and $\mathscr{C}_{i, \omega}^{k}$ equally hold.

Corollary 5.7. The natural map of the quotient spaces $\mathscr{S}_{i, \sigma}^{k} / \mathscr{D}_{\sigma} \rightarrow \mathscr{M}_{\omega}^{k}$ are homeomorphisms onto open subsets for $\sigma \in \mathscr{C}_{i, \omega}$.

Proof of 5.6. Let $W^{k}$ be as in 5.3 and $\nu_{\sigma}^{k}$ the fiber over $\sigma$. Then a candidate for the slice is $\mathscr{S}^{k}:=\exp \left(W^{k} \cap \nu_{\sigma}^{k}\right)$. The isotropy group $\mathscr{D}_{\sigma}$ acts on $\nu^{k}$ isometrically, and exp is $\mathscr{D}_{\omega}^{k+1}$-equivariant. Thus $\mathscr{D}_{0}$ fixes $\mathscr{S}^{k}$ (as a set), which proves (i).

Next we show (iii): We use $V^{k+1}$ and $A^{k+1}$ as in the proof of 5.1 and use the section $\beta: V^{k+1} \longrightarrow A^{k+1} \longrightarrow \mathscr{D}_{\omega}^{k+1}$. We may assume that $\widetilde{W}^{k}:=\mu_{\omega}(\beta \times i d)\left(V^{k+1} \times \mathscr{S}^{k}\right)$ is contained in $N^{k}$. Then a continuous inverse of $\mu_{\omega}(\beta \times \mathrm{id}): V^{k+1} \times \mathscr{S}^{k} \rightarrow \widetilde{W}^{k}$ is given by

$$
\iota(\tau)=\left(\beta \rho \pi \exp ^{-1}(\tau), \mu_{\omega}\left(\left(\beta \rho \pi \exp ^{-1}(\tau)\right)^{-1}, \tau\right)\right.
$$

where $\pi: \nu^{k} \rightarrow O_{o}^{k}$ is the bundle projection and $\rho$ is the inverse of the diffeomorphism $\mathscr{D}_{\omega}^{k+1} / \mathscr{D}_{0} \rightarrow O_{\sigma}^{k}$.

The second statement follows directly from the properness of the group action after a reduction in size of $\mathscr{S}^{k_{0}}$ Otherwise there existed points $\sigma_{n}$ and $\tau_{n} \neq \sigma_{n}$ in $\mathscr{S}^{k}$ tending to $\sigma$ and $\phi_{n} \in \mathscr{D}_{\omega}^{k+1}$ with $\tau_{n}=\mu\left(\phi_{n}, \sigma_{n}\right)$, and $\phi_{n} \notin \mathscr{D}_{0}$. After passing to a subsequence, we may assume that 
$\phi_{n}$ converge to some $\phi \in \mathscr{D}_{\omega}^{k+1}$ by (3.3), which has to be contained in $\mathscr{D}_{\sigma}$. Thus $\phi_{n}{ }^{\circ} \mathscr{D}_{\sigma} \subset V^{k+1}$ for sufficiently large $n$, and then $\phi_{n}^{*} \sigma_{n}=\tau_{n}$ for $\sigma_{n}, \tau_{n} \in \mathscr{S}^{k}$, which is impossible by (iii).

As $\mathscr{D}_{\omega}^{k+1}$ fixes $\mathscr{C}_{i, \omega}^{k}$, the statements on $\mathscr{S}_{i}^{k}$ are clear.

The chosen approach using weak Riemannian metrics enables us to compare slices and group actions for various $k$ :

Proposition 5.8. Let $\sigma \in \mathscr{C}_{i, \omega}$ and $k \geqq k_{0}:=2 m+4$ 。

(i) The map $\mu_{\omega}(\beta \times i d): V^{k+1} \times \mathscr{S}_{\omega}^{k+r} \rightarrow \mathscr{C}_{a, \omega}^{k}$ is of class $C^{\prime}$ 。

(ii) Let $R^{k}=\mu_{\omega}(\beta \times i d)\left(V^{k+1} \times \mathscr{S}^{k}\right) \subset \mathscr{C}_{a, \omega}^{k}$. Then the restriciion of $\iota: R^{k} \rightarrow V^{k+1} \times \mathscr{S}^{k}$ to $\mathbb{R}^{k+r}$ is of class $C^{r}$ for all $r \geqq 0$ 。

(iii) $\mathscr{S}^{k_{0}} \cap \mathscr{C}_{a, \omega}$ satisfy the conditions of 5.5 on $\mathscr{P}^{k}$.

In view of (iii) above, we take in the sequel $\mathscr{S}^{k} \cap \mathscr{C}_{a, \omega}^{k}$ as $\mathscr{S}^{k}$ and set $\mathscr{S}=\bigcap_{k \geqq k_{0}} \mathscr{S}^{k}$ and $\mathscr{S}_{i}=\mathscr{S}_{i, \sigma}=\bigcap_{k \geqq k_{0}} \mathscr{S}_{i, \sigma \circ}^{k}$ Observe that $\mathscr{S}$ is a slice for the action of $\mathscr{D}_{\omega}$ on $\mathscr{C}_{a, \omega}$

Proof. The statement (i) follows from 5.4, (ii) follows from 2.3, and (iii) follows from the fact that the group inversion $\mathscr{D}^{k+r} \rightarrow \mathscr{D}^{k}$ is of class $C^{r}$ and that a similar property holds for $\exp ^{-1}(\mathrm{cf} .[\mathrm{KO}, \mathrm{OM}])$.

\section{The Moduli Space of Kähler Structures on a Compact Real Symplectic Manifold}

Let $(M, \omega)$ as before be a compact real symplectic manifold of dimension $2 m$. We are interested in the moduli space $\mathscr{M}_{\omega}$ of isomorphism classes of Kähler structures of class $C^{\infty}$ on $(M, \omega)$. We assume that $\mathscr{M}_{\omega} \neq \varnothing$. We describe this space by means of almost $\mathbb{K}$ ähler structures of class $H^{k}$. The moduli space $\mathscr{M}_{\omega}$ is the inverse limit $\lim \mathscr{M}_{\omega^{\circ}}^{k} \quad$ We endow $\mathscr{M}_{\omega}$ with the inverse limit topology。 The results $\overrightarrow{k>2 m+3}$ of section 3 imply that the natural maps $\mathscr{M}_{\omega} \rightarrow \mathscr{M}_{\omega}^{k}$ are embeddings and the following

Proposition 6.1 . The space $\mathscr{M}_{\omega}$ is Hausdorff. 
The analogous statements hold for the moduli space $\mathscr{M}_{a, \omega}$ of isomorphism classes of almost Kähler structures on $(M, \omega)$.

The structure of these spaces can be described in the framework of the ILH-category (inverse limits of Hilbert spaces) introduced by Omori $[\mathrm{OM}]$. We briefly review the concept.

A system $\left\{E^{k} ; k \in \mathbb{N}, k \geqq d\right\}$ for some $d \in \mathbb{N}$ is called a Sobolev-chain if every $E^{k}$ is a real Hilbert space, and $E^{k+1}$ is linearly and densely embedded in $E^{k}$.

Definition 6.2. Let $N$ be a topological space. Then $N$ is a strong $C^{\infty}$ ILH-manifold modelled on a Sobolev chain $\left\{E^{k}\right\}_{k \geqq d}$, if there exists a family $N^{k}, k \geqq d$, of $C^{\infty}$ Hilbert manifolds modelled on $E^{k}$, if the following conditions hold:

(i) $N^{k+1} \longrightarrow N^{k}$ is a continuous inclusion.

(ii) There exists a homeomorphism $h: N \rightarrow \underset{k \geq d}{\cap} N^{k}$, where the intersection is endowed with the inverse limit topology.

(iii) For any $x \in N$ there exists a coordinate neighborhood $U(h(x))$ $\subset N^{d}$ and a coordinate map $\phi_{d}: U(h(x)) \rightarrow E^{d}$ such that

$$
\phi_{d}\left(U(h(x)) \cap N^{k}\right) \subset E^{k} \quad \text { and } \quad \phi_{d} \mid\left(U(h(x)) \cap N_{k}\right.
$$

is a coordinate map for $N^{k}$ for all $k \geqq d$.

One can also define in a similar way strong $\mathbb{C}$-analytic ILHmanifolds and -spaces modelled on a chain of complex Hilbert manifolds and spaces. For the definition of strong ILH-groups we refer to Omori [OM 1.2.1].

The groups of $H^{k}$-diffeomorphisms $\mathscr{D}^{k}$ of $M, k \geqq m+1$, together with their common intersection $\mathscr{D}$ are known to form a strong ILHgroup, and the group $\mathscr{D}_{\omega}$ of symplectic diffeomorphisms forms a closed ILH-subgroup of $\mathscr{D}$ [OM].

As a first remark we note that $\mathscr{C}_{a, \omega}$ is a strong $\mathbb{C}$-analytic ILHmanifold, containing the $\mathbb{C}$-analytic ILH-space $\mathscr{C}_{i, \omega}$. The action of the strong ILH-group $\mathscr{D}_{\omega}$ is continuous. For a description of the quotients we use the results of section 5. From Corollary 5.7, we obtain immediately the following

Proposition 6. $\mathfrak{B}_{\text {。 }}$ The topology of $\mathscr{M}_{\omega}$ is generated by all sets $\mathscr{S}_{i, \sigma} / \mathscr{D}_{0}$ 
where $\sigma \in \mathscr{C}_{i, \omega}$.

For those Kähler structures which allow no non-vanishing holomorphic vector fields one can say more: Let $\mathscr{C}_{i, \omega}^{k \prime} \subset \mathscr{C}_{i, \omega}^{k}$ denote the space of such structures. Then it is $\mathscr{D}_{\omega}^{k+1}$-invariant. $\mathscr{M}_{\omega}^{k}$ shall denote the quotient $\mathscr{C}_{i, \omega}^{k \prime} / \mathscr{D}_{\omega}^{k+1}, k \leqq \infty$, and we write $\mathscr{M}_{\omega}^{\prime}:=\mathscr{M}_{\omega}^{\prime \infty}$. In this situation all stabilizer groups $\mathscr{D}_{o}, \sigma \in \mathscr{C}_{i, \omega}^{k^{\prime}}$ are finite.

Lemma 6.4. The moduli space $\mathscr{M}_{\omega}^{\prime}$ is an open subspace of $\mathscr{M}_{\omega}$.

Proof. We show that the complement $\mathscr{A}:=\mathscr{C}_{i, \omega}^{k}-\mathscr{C}_{i, \omega}^{k \prime}$ is a closed analytic subspace. The proof of Kuranishi's theorem [KU] shows that integrable almost complex structures which are "close" to a given one $\tau$ in $\mathscr{C}_{i, \omega}^{k}$ are also close to $\tau$ in the sense of deformation theory and that $\mathscr{C}_{i}^{k}$ is locally a product of a finite dimensional analytic space $S$ which is the base of a versal deformation and an infinite dimensional manifold $\widetilde{D}$. However in the set of all points whose fibers possess holomorphic vector fields $\neq 0$ is an analytic set $A$, and $\mathscr{A}=A \times \widetilde{\mathscr{D}}$.

In order to describe the structure of $\mathscr{M}_{\omega}^{\prime}$, we introduce the following terminology:

Definition 6.5. (1) Let $N_{1}$ and $N_{2}$ be two strong $C^{\infty}$ ILHmanifolds modelled on Sobolev chains $\left\{E_{1}^{k}\right\}$ and $\left\{E_{2}^{k}\right\}$ respectively. $\mathbb{A}$ continuous map $f: N_{1} \rightarrow N_{2}$ is called a smooth $\mathbb{I L H}-m a p$, if the following condition is satisfied: For any $x \in N_{1}$ there exist neighborboods $U_{1}$ and $U_{2}$ of $x$ and $f(x)$ resp. with coordinate maps $\phi_{j}: U_{j} \rightarrow V_{j} \subset E, j=1,2$, such that $\tilde{f}:=\phi_{2} f \phi_{1}^{-1}$ extends to a continuous map $\tilde{f}^{k}: V_{1}^{k} \rightarrow V_{2}^{k}$ for each $k$, and its restriction $\tilde{f}^{k}: V_{1}^{k+r} \rightarrow V_{2}^{k}$ is of class $C^{r}$ for any $r \geqq 0$, where $V_{j}^{k} \subset E^{k}$ are open subsets associated to coordinate maps $\psi_{\text {。 }}$

(2) Let $N$ be a topological space. We call $N$ a strong $C^{\infty, 0} \mathbb{I L H}$ manifold modelled on a Sobolev chain $\left\{E^{k}\right\}$, if there exist some open covering $\left\{U_{i}\right\}$ of $N$, and on each $U_{i}$ a structure of a strong $C^{\infty} \mathbb{I L H}$ manifold modelled on $\left\{E^{k}\right\}$ such that if $U_{i j}:=U_{i} \cap U_{j} \neq \phi$ the identity of $U_{i j}$ is a smooth $\mathbb{I L H}$-map of one structure to the other in the sense of (1).

(3) Let $N$ be a strong $C^{\infty} \mathbb{L} \mathbb{H}$-manifold with a defining system 
of Hilbert manifolds $\left\{N^{k}\right\}$ and $K \subset N$ a closed subspace. Then $K$ is called a $C^{\infty} \mathrm{ILH}$-subspace, if the following condition is satisfied: There exist a strong $C^{\infty} \mathbb{L H}$-submanifold $A \subset \mathrm{N}$ and a strong $C$-analytic ILH-subspace $B \subset N$ defined by systems of closed $C^{\infty}$ submanifolds $A^{k} \subset N^{k}$ and closed $\mathbb{C}$-analytic subspaces $B^{k} \subset N^{k}$ resp. such that $K$ is the intersection of all $K^{k}=A^{k} \cap B^{k}$ with the inverse limit topology。

(4) Using (2) and (3), we can define the notion of a $C^{\infty, 0}$ subspace of a strong $C^{\infty, 0} \mathbb{L L H}$-manifold.

Example 6.6。 Denote by $\mathscr{R}, \mathscr{S}, V$ the ILH-manifolds induced by the inverse systems $\left\{\mathscr{R}^{k}\right\},\left\{\mathscr{S}^{k}\right\},\left\{V^{k}\right\}$ according to 5.8 (cf. also the proof). Then the following are examples of smooth $\mathbb{I L H}$-maps:

(1) The group inversion $\mathscr{D}_{\omega} \rightarrow \mathscr{D}_{\omega}$ (cf. [OM]).

(2) The action $\mu_{\omega}: \mathscr{D}_{\omega} \times \mathscr{C}_{a, \omega} \rightarrow \mathscr{C}_{a, \omega}$, in particular its restriction $\mu_{\omega}: V \times \mathscr{S}_{a, \omega} \rightarrow \mathscr{R}$.

(3) The inverse $h: \mathscr{R} \rightarrow V \times \mathscr{S}_{a, \omega}$ of $\mu \mid V \times \mathscr{S}_{a, \omega}$

Definition 6.\% Let $X$ be a topological Hausdorff space and $\bar{U}$ an open subset of $X$. Then a local ILH-uniformizing system of $\bar{U}$ is a quadrupel $(\tilde{U}, U, \mathscr{D}, \pi)$ consisting of a strong ILH-manifold $\tilde{U}$ modelled on a Sobolev chain $\left\{E^{k}\right\}$, a closed $C^{\infty}$ ILH-supspace $U$ of $\tilde{U}$, a finite group $\mathscr{D}$ which acts on $\tilde{U}$ smoothly and fixes $U$, and finally of a $\mathscr{D}$-equivariant continuous map $\pi: U \rightarrow \bar{U}$ which induces a homeomorphism $U / \mathscr{D} \rightarrow \bar{U}$, where $\mathscr{D}$ acts trivially on $\bar{U}$.

Definition 6.8. An ILH-V-space of class $C^{\infty, 0}$ consists of a topological Hausdorff space $X$ together with a collection $\mathscr{F}=\{\tilde{U}, U, \mathscr{D}, \pi)\}$ of local ILH-uniformizing systems such that all $\bar{U}$ generate the topology of $X$ and the following compatibility condition holds: If $(\tilde{U}, U, \mathscr{D}, \pi)$ and $\left(\tilde{U}^{\prime}, U^{\prime}, \mathscr{D}^{\prime}, \pi^{\prime}\right)$ are in $\mathscr{F}$ with $\bar{U} \subset \bar{U}^{\prime}$ then there exist a representation $\mathscr{D} \rightarrow \mathscr{D}^{\prime}$ and a $\left(\mathscr{D}, \mathscr{D}^{\prime}\right)$-equivariant smooth ILH-map of a neighborhood of $U$ in $\tilde{U}$ to $\tilde{U}^{\prime}$ which induces the embedding $\bar{U} \longrightarrow \bar{U}^{\prime}$. An ILH-V-manifold of class $C^{\infty, 0}$ is an ILH-Vspace, where one can choose local uniformizing systems with $\tilde{U}=U$.

Theorem 6.9. The moduli space $\mathscr{M}_{\omega}^{\prime}$ of Kähler structures on a real symplectic manifold $(M, \omega)$ that allow no holomorphic vector fields other than zero is a Hausdorff ILH-V-space of class $C^{\infty, 0}$. 
Proof. Let $\sigma \in \mathscr{C}_{i, \omega}^{\prime}$ and $\mathscr{S}_{\sigma}, \mathscr{S}_{\sigma, i}$ the slices of the action of $\mathscr{D}_{\omega}$ on $\mathscr{C}_{a, \omega}$ and $\mathscr{C}_{i, \omega}$ respectively. Let $U_{\sigma}=\mathscr{S}_{\sigma, i} / \mathscr{D}_{\sigma} \subset \mathscr{M}_{\omega}^{\prime}$. Then $\left(\mathscr{S}_{\sigma}, \mathscr{S}_{\sigma, i}, \mathscr{D}_{\sigma}\right.$, $\left.\pi_{\sigma}: \mathscr{S}_{\sigma, i} \rightarrow U_{\sigma}\right)$ is a local ILH-uniformizing system for $U_{\sigma}$. The construction of the $\mathscr{S}_{\sigma}$ allows us to assume that these are simply connected. We let $\sigma$ run through all points of $\mathscr{C}_{i, \omega}^{\infty}$ and $U_{\sigma}$ through a base of neighborhoods of the image of $\sigma$ in $\mathscr{M}_{\omega}^{\prime}$.

Let $\tau \in \mathscr{C}_{i, \omega}^{\prime}$ and $\left(\mathscr{S}_{\tau}, \mathscr{S}_{\tau, i}, \mathscr{D}_{\tau}, \pi_{\tau}: \mathscr{S}_{\tau, i} \rightarrow U_{\tau}\right)$ be a further uniformizing system such that $U_{\tau}$ is contained in $U_{\sigma}$. We have to show that there exists a $\left(\mathscr{D}_{\tau}, \mathscr{D}_{\sigma}\right)$-equivariant smooth ILH-map $\mathscr{S}_{\tau} \rightarrow \mathscr{S}_{\sigma}$. Let $\rho \in \mathscr{S}_{\tau}$ be any element. Then there exists a $\phi \in \mathscr{D}_{\omega}$ and a neighborhood $V(\rho) \subset \mathscr{S}_{\tau}$ such that $\phi^{*} \rho \in \mathscr{S}_{\sigma}$ and $\phi^{*} V(\rho) \subset \mathscr{R}$ (cf. 6.6). Then $\mu_{\omega}^{-1} \phi^{*}$ composed with the projection to $\mathscr{S}_{\sigma}$ gives a smooth ILH-map $V(\rho) \rightarrow \mathscr{S}_{\text {o }}$ which induces the embedding $\pi_{\tau}(V(\rho)) \longrightarrow U_{\sigma}$ (cf. 6.6). From this and the fact that $\mathscr{S}_{\tau}$ is simply connected, we can construct a smooth ILH-map $\lambda_{0}: \mathscr{S}_{\tau} \rightarrow \mathscr{S}_{\sigma}$ inducing $U_{\sigma} \rightarrow U_{\tau}$ such that $\lambda_{0} \mid V(\rho)$ is of the above kind. Then such a map $\lambda_{0}$ is unique up to the action of $\mathscr{D}_{\tau}$. Now $\lambda_{0}$ is certainly $\left(\mathscr{D}_{\tau}, \mathscr{D}_{\sigma}\right)$-equivariant.

\section{Relations Between $\mathscr{M}_{\omega}$ and the Moduli Space of Polarized Kähler Manifolds}

Let $(M, \omega, \sigma)$ be a symplectic manifold equipped with a Kähler structure $\sigma$. Then by the assignment $\omega \mapsto \lambda:=[\omega] \in H^{2}(M, \mathbb{R})$ it determines a polarized manifold $(X, \lambda)$ where $X=(M, \sigma)$. Let $\mathscr{M}_{\lambda}$ be the moduli space of all non-uniruled polarized Kähler manifolds with fixed Kähler class $\lambda ; \mathscr{M}_{\lambda}$ is an analytic space by [FUl, SGH]. Let $\mathscr{M}_{\omega}^{k^{\prime \prime}} \subset \mathscr{M}_{\omega}^{k}$ and $\mathscr{M}_{\omega}^{\prime \prime} \subset \mathscr{M}_{\omega}$ be the subspaces consisting of those Kähler structures $(M, \omega, \sigma)$ such that $(M, \sigma)$ is not uniruled. Then by the deformation invariance of the non-uniruledness [FU2] as in the proof of 6.4 one sees that $\mathscr{M}_{\omega}^{\prime \prime} \subset \mathscr{M}_{\omega}$ is open. We denote the canonical maps by $\Pi_{\omega}^{k}: \mathscr{M}_{\omega}^{k \prime \prime} \rightarrow \mathscr{M}_{\lambda} ; \Pi_{\omega}: \mathscr{M}_{\omega}^{\prime \prime} \rightarrow \mathscr{M}_{\lambda}$.

Theorem 7.1. Let $(M, \omega)$ be a symplectic manifold. Then for all $k>\operatorname{dim}(M)+3$ and $\lambda=[\omega]$ the images of the canonical maps $\Pi_{\omega}^{k}: \mathscr{M}_{\omega}^{k \prime \prime} \rightarrow \mathscr{M}_{\lambda}$ are open and closed. In particular, the image of $\Pi_{\omega}^{\infty}: \mathscr{M}_{\omega}^{\prime \prime} \rightarrow \mathscr{M}_{\lambda}$ is open and closed. 
Proof. Let $\left(M, \sigma_{0}, \omega\right)$ be a Kähler manifod, $\sigma_{0}$ of class $H^{k}$ and $p \in \mathscr{M}_{\lambda}$ the isomorphism class of the induced polarized manifold $\left(X_{0}, \lambda_{0}\right)$. Let $\left(\mathscr{X} \rightarrow S, \lambda_{\mathscr{X} / S}\right)$ be a local universal family whose distinguished fiber $\left(X_{s_{0}}, \lambda_{X_{s_{0}}}\right)$ is endowed with a fixed isomorphism with $\left(X_{0} ; \lambda_{0}\right)$. The natural map from $S$ to $\mathscr{M}_{\lambda}$ sending $s \in S$ to the isomorphism class of the fiber $\left(X_{s}, \lambda_{X_{s}}\right)$ is holomorphic. Its image is an open neighborhood of $p$. The openness of $\Pi_{\omega}^{k}$ is proved if we show for all $s \in S$ the existence of a $\sigma \in \mathscr{C}_{i, \omega}^{k}$ such that $((M, \sigma), \lambda)$ is isomorphic to $\left(X_{s}, \lambda_{X_{s}}\right)$. For $s=s_{0}$ this is true. We connect any point $s \in S$ with $s_{0}$ by a real differentiable $\operatorname{arc} \gamma(t), t \in[0,1]$. As the polarization $\lambda_{\mathscr{X} / S}$ can be realized by a relative Kähler form $\omega_{\mathscr{X} / S}$, where $\omega_{s}:=\omega_{\mathscr{X} / S} \mid X_{s}$ equals $\omega$ for $s=s_{0}$, we have a family of symplectic forms $\eta_{t}=\omega_{\gamma(t)}$ on $M$ with $\left[\eta_{t}\right]=\lambda_{0}$ By a theorem of Moser [MO] there exists a diffeomorphism $\phi$ on $M$ such that $\phi^{*} \omega_{s} \equiv \phi^{*} \eta_{1}=\omega$. This means that $\left(X_{s}, \lambda_{X_{s}}\right)$ can be realized by a Kähler structure on the symplectic manifold $(M, \omega)$. The closedness of the images of $\Pi_{\omega}^{k}$ follows from the proposition below.

Let $\lambda \in H^{2}(M, \mathbb{R})$ be fixed and $\Gamma_{\lambda}$ be the set of all $C^{\infty}$ symplectic

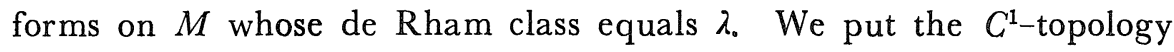
on $\Gamma_{\lambda}$. Let $\left\{\Gamma_{l}\right\}_{\iota \in I}$ be the set of connected components of $\Gamma_{\lambda}$ which

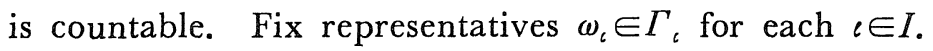

Proposition 7.2. The moduli space $\mathscr{M}_{\lambda}$ is the union of all $\Pi_{\omega_{\iota}}^{k}\left(\mathscr{M}_{\omega_{\iota}}^{k}\right)$, $\iota \in I$. The images of any two $\mathscr{M}_{\omega_{\iota}}^{k}$ and $\mathscr{M}_{\omega_{\kappa}}^{k}$ are disjoint or equal.

Proof. If $\omega_{1}, \omega_{2}$ are in the same connected component of $\Gamma_{\lambda}$, then by the above theorem of Moser, there is a $C^{\infty}$-diffeomorphism $\phi$ of $M$ such that $\phi^{*} \omega_{2}=\omega_{1}$. This implies that the images of $\mathscr{M}_{\omega_{1}}^{\prime \prime}$ and $\mathscr{M}_{\omega_{2}}^{\prime \prime}$ are the same. The first assertion follows from this and the definition.

For the second assertion let $(M, \sigma, \omega)$ and $(M, \tau, \tilde{\omega})$ be Kähler structures, and $\psi:(M ; \sigma),[\omega]) \rightarrow((M, \tau),[\tilde{\omega}])$ an isomorphism of polarized manifolds, in particular $\psi^{*}[\tilde{\omega}]=[\omega]$. The family $\omega_{t}=t \cdot \omega$ $+(1-t) \cdot \psi^{*} \tilde{\omega}$ consists of Kähler forms with respect to $\sigma$ in a fixed cohomology class. Again, Moser's theorem gives a diffeomorphism $\chi: M \rightarrow M$ such that $\chi^{*} \phi^{*} \tilde{\omega}=\omega$. Now by means of the map $\phi \chi$ the connected components of $\mathscr{M}_{\omega}^{k}$ and $\mathscr{M}_{\tilde{\omega}}^{k}$ containing $(M, \sigma, \omega)$ and $(M, \tau, \tilde{\omega})$ 
resp. are transformed bijectively into each other. So the images under $\Pi_{\omega}^{k}$ and $\Pi_{\tilde{\omega}}^{k}$ are equal.

Theorem 7.3. Let $(M, \lambda)$ be a compact differentiable manifold with $\lambda \in H^{2}(M, \mathbb{R})$. Then the moduli space $\mathscr{M}_{\lambda}$ of polarized Kähler structures on $(M, \lambda)$ has countable topology.

Proof. From the second section we know that all $\mathscr{C}_{a, \omega}^{k} \subset U(\sigma) \subset$ $H^{k}\left(S_{0}\right)$ are Hilbert-analytic manifolds containing the closed Hilbertanalytic subspaces $\mathscr{C}_{i, \omega}^{k}$. These consist of countably many components with countable topology. The subspaces of complex structures which allow non-zero holomorphic vector fields or yield uniruled manifolds are closed analytic subspaces. Since the moduli spaces $\mathscr{M}_{\omega}^{k}$ carry the quotient topology, these have a countable topology as well. Furthermore the topology on the inverse limit $\mathscr{M}_{\omega}=\lim \mathscr{M}_{\omega}^{k}$ is countable. Now

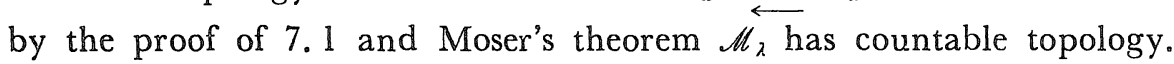

\section{References}

[BE-EB] Berger, M. and Ebin, D., Some decompositions of the space of symmetric tensors on a Riemannian manifold, J. Diff. Geom., 3 (1969), 379-392.

[D-N] Douglis, A. and Nirenberg, L., Interior estimates for elliptic systems of partial differential equations. Comm. pure appl. math., 8(1955), 503-538.

[DOU] Douady, A., Le problème des modules pour les variétés analytiques complexes, Séminaire Bourbaki, 1964/1965, No 277.

[EB] Ebin, D., The manifold of Riemannian metrics, Global Analysis, Berkeley 1968, Proc. Symp. Pure Math. AMS, 15 (1970), 11-40.

[FU1] Fujiki, A., Deformations of uniruled manifolds, Publ. RIMS, Kyoto Univ., 17 (1981), 687-702.

[FU2] - Coarse moduli space for polarized compact Kảhler manifolds, Publ. RIMS, Kyoto Univ., 20 (1984), 977-1005.

[G-T] Gilbarg, D. and Trudinger, N.S., Elliptic partial differential equations of second order, (Springer Verlag, Berlin, Heidelberg, New York, 1977).

[K-N] Kobayashi, S. and Nomizu, K., Foundations of differential geometry, vol I, II, (Interscience Publishers, New York, London, Sydney, 1963, 1969.)

[KO] Koiso, N., Non-deformability of Einstein metrics, Osaka J. Math., 15(1978), 419-433.

[KU] Kuranishi, M., New proof for the existence of locally complete families of complex structures, Proc. Conference on Complex Analysis, Minneapolis 1964, (Springer Verlag, Heidelberg 1965, pp. 142-154).

[MO] Moser, J., On the volume elements of a manifold, Trans. Amer. Math. Soc., 120 (1965), 286-294.

[OM] Omori, H., Infinite dimensional Lie transformation groups, Lecture Notes in Mathematics 427, (Springer Verlag, Berlin, Heidelberg, New York, 1974).

[PA] Palais, R., Foundations of Global Analysis, (Benjamin, New York, Amsterdam, 1968). 
[SCH1] Schumacher, G., Construction of the coarse moduli space of compact polarized Kähler manifolds with $c_{1}=0$, Math. Ann., 264 (1983), 81-90.

[SCH2] - Moduli of polarized Kähler manifolds, Math. Ann., 269 (1984), 137-144.

[YA] Yano, K., Differential geometry on complex and almost complex spaces, (Pergamon Press, New York, 1965). 